INRA Prod. Anim.

2012, 25 (2), 85-100

\title{
Vingt ans de recherche-développement sur le travail en élevage : acquis et perspectives
}

\author{
B. DEDIEU ${ }^{1,2,3,4}$, G. SERVIÈRE 5
}

${ }^{1}$ INRA, UMR1273 Métafort, F-63122 Saint-Genès-Champanelle, France

2 AgroParisTech, UMR Métafort, BP 90054, F-63172 Aubière, France

${ }^{3}$ Irstea, UMR Métafort, BP 50085, F-63172 Aubière, France

${ }^{4}$ Clermont Université, VetAgro Sup, UMR Métafort, BP 35, F-63370 Lempdes, France

5 Institut de l'Elevage, Cité régionale de l'Agriculture, 9 allée Pierre de Fermat, F-63170 Aubière, France

Courriel :dedieu@clermont.inra.fr

La prise en compte du travail est essentielle pour l'analyse des systèmes d'élevage d'herbivores et de granivores, tant pour comprendre leurs fonctionnements et leurs transformations actuelles que pour se projeter vers l'avenir. C'est un défi pour la zootechnie, car les choix techniques impactent fortement le travail. Pour le partenariat recherche, formation et développement, il s'agit de renouveler les postures ainsi que les contenus du conseil aux éleveurs et de l'enseignement.

Cela fait vingt ans que la zootechnie des systèmes d'élevage intègre la dimension du travail dans l'analyse du fonctionnement et de la dynamique des systèmes d'élevage, et que cette discipline investit un domaine a priori réservé aux sciences sociales. La sociologie, la gestion, la psychologie, l'économie et l'ergonomie peuvent revendiquer une antériorité et une production scientifique abondante sur différentes déclinaisons du «travail», y compris en élevage (Beguin et al 2011). En effet, pour ces disciplines, l'élevage présente l'originalité d'être mis en œuvre selon des modèles de production très différents. Il est emblématique à la fois $i$ ) des savoirs de nature, de l'animal et du troupeau (Salmona 1994, Meuret 2010, Porcher 2011), de la persistance de formes traditionnelles de production fondées sur l'utilisation de ressources naturelles, ii) de l'agriculture familiale ; l'élevage laitier est ainsi emblématique de l'agriculture de couple (Remy 2008) et iii) de la modernité avec le développement d'un élevage, notamment de monogastriques, industrialisé tant par la prégnance des normes techniques produites par la recherche et l'aval (Nicourt et Cabaret 2011) que par les modes de division du travail entre éleveurs et salariés (Depoudent et Le Moan 2011).

Les zootechniciens ont pourtant investi la dimension du travail dans l'analyse des systèmes d'élevage. En suggérant des changements dans la condui- te des troupeaux et des surfaces, en proposant des techniques innovantes dans un souci d'amélioration de l'efficience technico-économique et/ou environnementale des systèmes de production, le zootechnicien modifie le travail à réaliser, la durée, les rythmes ou la possibilité de différer des tâches, les compétences et savoir-faire requis, les équipes de travail. Par exemple, l'ensilage de maïs, pilier de la révolution verte, définit à la fois une date de récolte spécifique, un chantier sollicitant des hommes et des machines en nombre pour une exécution rapide, et un mode de distribution du fourrage en bâtiment. Les changements techniques ont ainsi un impact fort à la fois sur le travail à faire, sur les personnes et les équipements pour le faire. La période récente de modernisation de l'agriculture est caractérisée par l'augmentation régulière des dimensions des exploitations et la forte diminution de la main-d'œuvre. Ces tendances influent sur l'adoption de paquets technologiques (Mak 2001) et mettent en question les modalités du travail avec les animaux, constitutives du métier, de la «passion des bêtes» (Soriano 1985).

Jusque dans les années 1980, la zootechnie, dans sa construction comme discipline, n'avait pas proposé de regard sur le travail tel qu'il est organisé et réalisé, ni sur l'homme au travail, notamment avec les animaux. Depuis, l'éthologie animale et la zootechnie des systèmes d'élevage ont largement inves- ti ces sujets. L'éthologie animale considère les interactions homme/éleveuranimaux et leurs conséquences sur le bien-être animal (Boivin et al 2012). La zootechnie des systèmes d'élevage a pour objet d'étude le fonctionnement et la dynamique d'un système constitué d'un pôle humain (l'éleveur, ses décisions et ses pratiques) et d'un pôle composé du troupeau et des ressources (élaboration de la production et renouvellement) (Dedieu et al 2008a). La zootechnie de l'animal, par ses connaissances sur les capacités adaptatives des animaux, contribue également à l'allègement et à la simplification des charges de travail des éleveurs (voir les articles de Hostiou et Fagon (2012) et d'Agabriel et al (2012) dans ce numéro).

Nous centrons cet article sur la contribution spécifique de la zootechnie des systèmes d'élevage, dont l'intérêt pour les questions de travail des éleveurs date de la fin des années 1980. Cette contribution a trois facettes : la construction d'un cadre d'analyse dans le champ de la discipline, la production de méthodes et outils opérationnels utilisables par des chercheurs et agents de développement, l'émergence d'une communauté recherche - formation - développement sur ce thème du travail en élevage. Nous évoquons dans une première partie les mutations du travail qui caractérisent l'évolution de l'agriculture ainsi que les enjeux d'adaptation des systèmes et des conduites d'élevage qui leur sont 
associés. Puis, en faisant référence aux points de vue de sciences sociales que nous avons pris en compte, nous présentons les bases conceptuelles des modèles du travail développés par les zootechniciens. Enfin, nous insistons sur les formes et les produits du partenariat scientifique et professionnel qui ont permis l'émergence progressive de cette communauté recherche - formation développement sur le travail en élevage.

\section{$1 /$ Les mutations du travail}

Parler de mutations du travail c'est envisager un ensemble d'évolutions structurelles, techniques et sociologiques qui modifient tout à la fois les conditions de travail (la vision objective) mais aussi les conditions de vie au travail (la vision subjective).

\section{1 / Les évolutions structurelles}

La diminution du nombre d'actifs agricoles, débutée dès la fin du

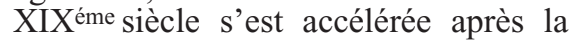
deuxième guerre mondiale. Dans la dernière décennie (2000-2010), le nombre d'Unité de Travail Annuel (UTA) a diminué de $22 \%$. En 1980, la population active agricole représentait encore $8 \%$ de la population active totale et seulement 3\% en 2008 (Agreste 2008). Il en est de même du nombre d'exploitations agricoles, qui est passé de 1017000 exploitations en 1988 à 490000 au dernier recensement (2010) tandis que la superficie moyenne de la ferme française passait de 31 ha à 55 ha. L'orientation bovin lait, comparativement aux bovins à viande et aux granivores, a été particulièrement affectée avec la disparition d'un tiers des exploitations en dix ans. Ainsi la productivité apparente du travail exprimé en ha de Surface Agricole Utile (SAU) par UTA s'est accrue dans des proportions considérables à un rythme compris entre 3 et $4 \%$ par an depuis les années 1980. Si l'on inclut les gains de productivité techniques (rendements à l'hectare ou à l'animal), la valeur ajoutée brute par actif a été multipliée par quatre sur les trente dernières années. Comparativement à l'ensemble de l'économie nationale, elle a évolué deux fois plus rapidement. L'accroissement de la productivité du travail est une des conditions de la compétitivité des entreprises agricoles, mais pour maintenir (en termes réels) le revenu agricole, l'augmentation des subventions s'est avérée indispensable (Charroin et al 2012).

L'évolution de la main-d'œuvre agricole est différenciée selon les catégories. La part des conjoints non coexploitants et aides familiaux est passée de près de $30 \%$ des UTA en 1988 à $15 \%$ en 2005. Cette tendance s'accentue; ainsi $75 \%$ des conjoints d'exploitants agricoles de moins de 30 ans ne travaillent jamais sur l'exploitation (contre $35 \%$ pour ceux de plus de 50 ans). Le statut des femmes évolue, d' «aide familiale» ou «conjoint participant aux travaux», elles deviennent plutôt «coexploitant» ou «conjoint collaborateur». Leurs installations sont aussi plus fréquentes $(22 \%$ des installations individuelles aidées en 2009). Corrélativement la part des UTA salariées permanentes est passée de 16\% (1988) à $29 \%$ (2007). En plus d'une internalisation du salariat au sein des exploitations, une externalisation plus importante de tâches, via l'entreprise de travail agricole ou la Coopérative d'Utilisation du Matériel Agricole (CUMA) se développe. Dans la filière bovine laitière, elle représente maintenant $13 \%$ des UTA totales. La croissance du nombre de groupements d'employeurs (forme d'emploi partagé entre agriculteurs, ou avec d'autres acteurs économiques), qui atteint 3700 pour 18 000 équivalents temps plein, contribue à l'évolution du contour de l'exploitation agricole. En même temps, la perméabilité entre catégories socioprofessionnelles progresse, de nombreux jeunes agriculteurs ont été salariés avant de s'installer et certains agriculteurs en difficulté peuvent trouver un emploi par exemple dans des groupements d'employeurs (Chabanet et al 1999).

L'agrandissement, le plus souvent accompagné de la spécialisation, n'a jamais été le seul trait d'évolution des exploitations. Dans le secteur agricole, la part de chefs d'exploitation pluriactifs, c'est à dire exerçant une autre activité en plus de l'activité agricole, augmente légèrement depuis 1988 et dépasse maintenant les $22 \%$. La pluriactivité des ménages agricoles a de son côté explosée : en 2000 , plus de $50 \%$ des conjoints travaillent à l'extérieur contre un tiers en 1988.

Le modèle d'exploitation familiale gérée par un couple et porté par la loi d'orientation agricole de 1962 n'est plus de mise. Le caractère familial de l'activité agricole demeure très prégnant (Brookfield 2008) mais a changé de nature (Dufour et Giraud 2012) du fait des complexifications de la composition de la main-d'œuvre, des systèmes d'activités des ménages agricoles (Laurent et al 1998) et des statuts juridiques des exploitations. Les formes sociétaires (GAEC: Groupement Agricole d'Exploitations en Commun, EARL : Exploitation Agricole à Responsabilité Limitée) ont augmenté de $3,9 \%$ par an depuis 1988 pour atteindre $28 \%$ de l'ensemble des exploitations. Le partage des responsabilités, du travail et des capitaux nécessite le passage de relations où l'implicite peut trouver sa place (dans les relations familiales) à des relations où il est nécessaire de s'expliquer, surtout lorsque les membres du collectif de main-d'œuvre n'ont aucun lien de parenté.

Ces tendances d'évolution de l'agriculture en général et de l'élevage en particulier sont partagées au niveau européen et pour l'ensemble des pays de l'OCDE. L'Allemagne a perdu $36 \%$ de ses exploitations ces dix dernières années, l'Italie 24\% (Agreste 2011). L'accroissement continu de la productivité du travail résulte de l'agrandissement des dimensions par travailleur et, surtout pendant les trente glorieuses, de l'augmentation des rendements par unité de surface ou par animal. Le modèle entrepreneurial capitalisé trouve à s'y développer comme voie modernisée de la ferme familiale.

En revanche, dans la plus grande partie des autres pays du monde (Asie, Afrique notamment), l'agrandissement des surfaces par travailleur a été quasi nul ces vingt dernières années, la pression foncière sur les terres demeurant très forte (Paillard et al 2011). Lagriculture familiale peu capitalisée demeure dominante dans ces régions, sans que l'on puisse exclure y compris dans des petites fermes, le recours temporaire à du salariat (Hostiou et al 2010). En Amérique du Sud, un nouveau modèle agricole voit le jour, celui d'une agriculture de fonds d'investissements (y compris de «feedlots») qui va sans doute, si l'intérêt que porte le monde de la finance à l'agriculture se confirme, modifier radicalement la diversité des systèmes, du travail et de la main-d'œuvre (Gras et Hernandez 2007).

\section{2 / Les moteurs du changement technique}

Nous ne pouvons ici qu'effleurer la dynamique de rationalisation technique de l'élevage, à laquelle la recherche a beaucoup contribué, en relation avec trois moteurs principaux de changement: la Politique Agricole Commune (PAC), les filières et les marchés, la société. Ces moteurs amènent l'élevage à répondre à une demande globale de programmation des ventes, si possible mieux réparties sur l'année, avec des produits répondant à des spécifications de plus en plus précises et des pratiques devant mieux respecter l'environnement ou le bien-être animal. Ces moteurs sont assortis de propositions contractuelles, de réglementations nouvelles et donc de perspectives de contrôles. Ils accompagnent le mouvement d'accroissement de la productivité du travail évoqué ci-des- 
sus. Ces spécifications imposant un surcroît de travail matériel (Veron et Dobremez 2004), de tension mentale (Laurent et al 2000) et des enregistrements supplémentaires (Havet et al 2005), sont diversement reçues par les agriculteurs.

L'avenir se dessine autour de versions productives et écologisées de l'élevage, répondant aux défis de l'alimentation de la population mondiale en croissance et de la préservation de l'environnement (Coulon et Lecomte 2009). D'ores et déjà plusieurs modèles de cet élevage de demain apparaissent. L'un relève des perspectives offertes par «l'élevage de précision» (Lokhorst et Groot Koerkamp 2009) avec la sélection génomique, la robotisation (Billon et Pomiés 2006) et le développement de systèmes électroniques d'information sur différents paramètres physiologiques ou de carrière de l'animal. On est à l'aube de changements radicaux dans la façon de faire de l'élevage et dans le travail des agriculteurs notamment dans leurs rapports aux animaux et à l'information. L'agroécologie (Altieri 1992) et ses déclinaisons actuelles en France et en Europe (agriculture biologique, autonome, de proximité) constitue une autre voie d'évolution de l'élevage (plus pré-

Figure 1. Attentes des éleveurs bovins laitiers (392 exploitations, 452 éleveurs) (Seegers et al 2006).

\section{Le temps qu'il reste}

Pour assurer les autres travaux : entretien du matériel, comptabilité...

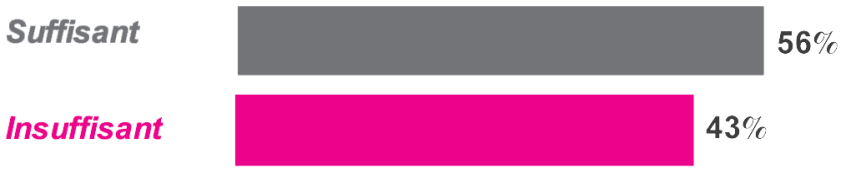

Pour mes loisirs, mes activités sociales...

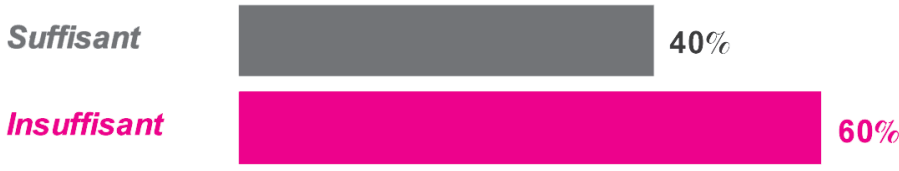

Les aspirations

Se libérer du temps au quotidien

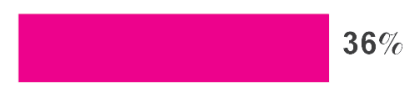

Se libérer plus souvent les week-onds

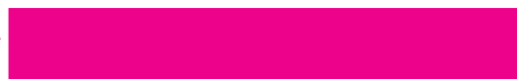

$61 \%$ encore les implications sont importantes sur la durée du travail, sa répartition dans le temps mais aussi sur son contenu, par exemple avec une surveillance accrue des animaux ou de nouvelles interactions avec les consommateurs 2011).

\section{3 / Evolutions des identités et rapport au travail}

Les agriculteurs ont changé dans leur perception du travail, signant la fin de labeur payarthez (1986) appelait «le vail étaient confondus. Aujourd'hui, la distinction est plus fréquente et les rapports des agriculteurs à leur travail sont contrastés. Ainsi, en élevage laitier du Ségala (Aveyron, Lot), Dufour et res :

- la passion «faut être passionné, parce que si on n'est pas passionné 'est pas la peine,... faut être passionne des bêtes, parce qu'il faut vraiment les uivre»; ducteur de lait et pour assumer ça bien dans le temps, il faut s'organiser sinon ce n'est pas possible». Cette figure cisément de la polyculture-élevage). Là montante de la modernité présente une facette tournée vers la productivité du travail, condition de la compétitivité, et une autre vers une articulation harmonieuse entre temps privés et temps professionnels ;

- le travail difficile "ron subit les animaux, on subit la terre, le biologique, on ne peut pas le maîtriser». L'amertume d'être à l'écart des normes de la société, y compris au sein de sa propre famille, est forte, le travail étant vécu comme écrasant.

Dans l'ensemble de ces registres le rapport au temps de travail est une donnée incontournable mais variable (Fiorelli et al 2010). Certains ne comptent pas leur temps (passion), d'autres déplorent une durée du travail excessive (difficile), alors que d'autres cherchent à se dégager du temps (au quotidien, le week-end, pour des congés) (Seegers et al 2006) (figure 1). Cette diversité soulignée par plusieurs auteurs (Salmona 1994, Blanchemanche 2000) montre que le travail, parce qu'il permet tout autant de produire que de se produire (Dejours 1998, Porcher, 2002), a des composantes subjectives fortes. Comme dans l'ensemble de la société, le besoin de reconnaissance et de réalisation individuelle dans le travail croît en agriculture (Fiorelli 2010), avec pour l'élevage cette dimension supplémentaire de l'affectif envers les animaux. Plusieurs auteurs interrogent alors certaines dynamiques de l'élevage (agrandissement, intégration, industrialisation) selon qu'elles permettent ou non l'expression de la subjectivité de chacun (Fiorelli et al 2012) et le développement ou non de sa capacité à produire ses propres normes de travail (Nicourt et Cabaret 2011).

\section{4 / Implications pour la recher- che}

L'ensemble des tendances résumées ci-dessus montre bien l'ampleur des mutations du travail agricole dans leurs composantes structurelles, techniques et sociologiques. Ces mutations sont porteuses d'enjeux de productivité, d'articulation entre activités agricole, non agricole et privée et de sens du travail (encadré 1). Elles s'expriment dans des exploitations hétérogènes, i) quant à la main-d'œuvre permanente (de l'agriculteur seul car le conjoint travaille à l'extérieur, aux GAEC avec de nombreux associés non familiaux ou à l'exploitant avec salariés en passant par la figure traditionnelle du couple), ii) quant à leur évolution selon des dynamiques d'agrandissement et/ou de diversification des activités, en filière longue ou courte, et iii) dans des systèmes de production très inégalement normalisés et réglementés. 
Encadré 1. Hypothèses d'évolution des systèmes de travail selon G. Rault (2005).

Trois orientations sont rencontrées :

1) une accentuation de la rationalisation du travail avec des références de plus en plus affirmées au monde industriel (calcul du temps de travail, délégation, salariat). Le travail agricole pourrait osciller entre une logique d'artisanat et de très petite entreprise de soustraitance.

2) la recherche d'une maîtrise sur l'exploitation avec en corollaire une volonté d'autonomie. Le projet de vie et le temps personnel deviennent centraux. Les thématiques sont la polyvalence, la compétence, l'articulation entre les sphères professionnelles et personnelles.

3) un renouveau de l'organisation clanique (familiale élargie), avec de nouvelles articulations entre logiques familiales et entrepreneuriales. Le travail agricole doit permettre le maintien du patrimoine familial et assurer d'abord une assise sociale et/ou économique à tous. La gestion de l'exploitation peut être au coup par coup.

La recherche agronomique a accompagné les mutations évoquées en proposant des paquets techniques ou des modèles susceptibles d'améliorer l'efficience des systèmes dans leur rapport à la production animale et à l'environnement. Dans ces propositions, l'homme est présent sous la forme implicite d'un acteur rationnel visant la maximisation du profit (Darnhofer et al 2009) ou d'un entrepreneur (Commandeur 2006). Cette approche continue de dominer la recherche en sciences animales, notamment en Europe. Les articles comportant une expression du travail sont quasi inexistants, sauf pour l'établissement de bilans technico-économiques incluant des équivalents travailleurs à temps complets («labour units») eux-mêmes assez rares. Les communautés scientifiques relevant des «farming systems» (Darnhofer et al 2012) ont proposé de rendre compte de l'agriculteur comme un manager mais aussi i) comme un être ayant ses propres perceptions du monde et des motivations personnelles, ii) comme un organisateur du travail et iii) comme un travailleur opérateur (Dedieu et Servière 2011). Dans cette communauté se discutent aujourd'hui les questions de travail en agriculture, autour notamment du concept de «liveability» (vivabilité) (IFSA 2010, Milestad et al 2012). Dans ce cadre, l'agronomie systémique développée par Sebillotte (1986) a été la première à proposer une vision plus élaborée de l'agriculteur, à la fois manager et organisateur du travail. Le logiciel «Otelo» développé avec les sciences de gestion (Attonaty et al 1990) a mis en relation dans le cas d'exploitations de grandes cultures, des journées disponibles, des règles de priorités entre travaux, de la main-d'œuvre, des équipements et des débits de chantiers. De même Cerf et Sagory (2004) ont pointé les différentes dimensions de l'agriculteur, entre manager, concepteur, organisateur et exécutant ainsi que le caractère fondamentalement aléatoire de l'activité qui implique de disposer de souplesses pour organiser le travail.

\section{2 / Les choix de conduite d'élevage : interactions avec le travail des éleveurs}

La conduite de l'élevage fait partie intégrante du «système travail», expression voulant rendre compte des multiples dimensions attachées à cette notion et à leurs interactions. Nous proposons ainsi tout d'abord le positionnement théorique permettant de relier les choix techniques, les tâches, le travail et son organisation, puis une série d'illustrations des modalités d'interactions entre conduite d'élevage et travail des éleveurs.

\section{1 / Positionnement théorique}

La conduite, vue comme un ensemble de décisions touchant l'élaboration de la production et le renouvellement des capacités productives du troupeau ainsi que des ressources est au cœur des questions de travail en élevage, au même titre que la dimension humaine, évidemment fondatrice de l'idée de travail. L'ergonomie de langue française distingue, parmi les fondements même de la discipline, le travail prescrit du travail réel (Leplat 1994). Le travail prescrit est normalisé par la technostructure, par les ingénieurs concepteurs des processus. Le travail réel n'existe que lorsque l'opérateur investit sa subjectivité, son expérience, ses compétences, ses astuces pratiques. Il transforme le travail prescrit parce que le travail est vivant, humain (Dejours 1998). Dans le monde de l'industrie, les fonctions de prescription du travail, d'organisation et celui des opérateurs de la production relèvent de groupes professionnels séparés (Mintzberg 2002), les premiers et les derniers étant souvent très peu associés dans le développement d'une innovation (Beguin 2011).

Dans l'exploitation agricole, l'éleveur est à la fois celui qui conçoit et pilote (Landais et Deffontaines 1988). Il est donc en mesure d'adapter tout autant la conduite et les artefacts technologiques que l'organisation de la main-d'œuvre et ses propres procédures de mise en œuvre du travail réel. La conduite de l'élevage est, de ce point de vue théorique, enchâssée dans un système complexe de travail (Madelrieux et al 2009). Le travail n'est donc pas seulement une ressource pour assurer un projet de production défini par un entrepreneur, ou à l'inverse, un facteur limitant de ce projet selon la vision développée par Lorino (1995) en sciences de gestion. Les questions de travail que se pose un agriculteur interfèrent avec la définition du projet de production animale, du corps de règles à mettre en œuvre pour orienter les processus de production et des procédures opérationnelles assurant leur mise en œuvre effective.

\section{2 / Les domaines de l'interac- tion entre conduite de l'élevage et travail des éleveurs}

Nous illustrons cette interaction entre conduite de l'élevage et travail des éleveurs dans quatre domaines. Deux domaines portent sur les implications des choix techniques sur la durée du travail et sur les représentations du métier. Deux domaines, à l'inverse, traitent des implications de pressions (volumes de travail important) ou d'attentes de travail (week-ends libérés, horaires maîtrisés) sur le pilotage du système et les procédures opérationnelles.

a) Les choix de conduite technique jouent sur la durée et la répartition du travail

Nous avons, avec l'exemple de l'ensilage de maïs, illustré les conséquences de cette technique sur le travail de récolte et de distribution. D'autres exemples peuvent être évoqués car ayant des impacts très forts sur la durée du travail. Par exemple, le gardiennage de troupeaux en milieu pastoral est très exigeant en temps (entre 3,5 et $11 \mathrm{~h}$ par jour selon les saisons en élevage ovin cévenol) (Dedieu 1984), ce qui justifie que des structures de développement encouragent la clôture des parcours. Des décisions de configuration des systèmes techniques comme le choix des périodes de mise bas ou de la durée d'hivernage en élevage bovin allaitant ont des implications fortes sur le profil d'évolution du travail d'astreinte sur l'année, la durée et l'intensité des périodes de pointe, et les concurrences avec le travail de saison (figure 2). En production porcine, le choix d'une conduite en bandes a des répercutions marquées sur l'organisation hebdomadaire du travail (Martel et al 2008). Cette relation forte entre les choix techniques et la durée du travail justifie l'effort de 
Figure 2. Deux profils d'évolution du travail d'astreinte en élevage bovin allaitant selon la distribution des vêlages au cours de l'année (Cournut et al 2009).

Profil plat : Pas de modification sensible du travail d'astreinte sur l'année. Cette exploitation des Pyrénées-Atlantiques se caractérise par des vêlages étalés toute l'année.

Profil en U : Rupture très marquée de la durée du travail d'astreinte quotidien avec une période hivernale qui est assez courte (4 à 5 mois). Cette exploitation du Limousin se caractérise par des vêlages groupés en automne et début d'hiver.

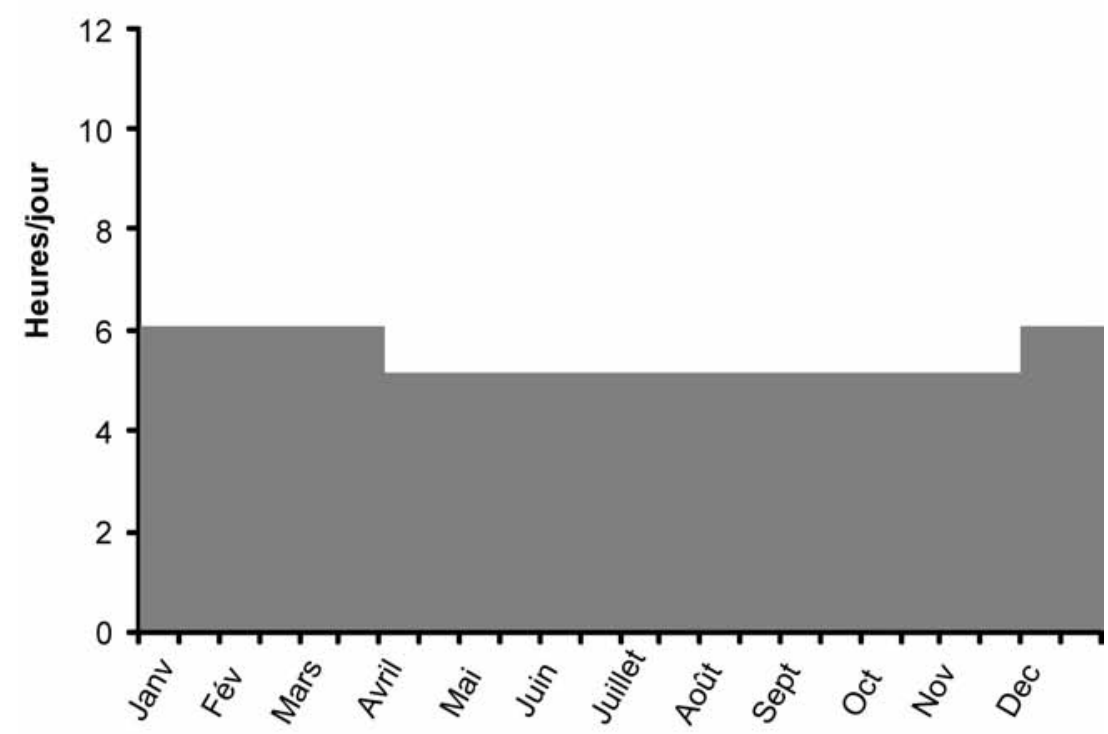

Profil plat «pas de modification»

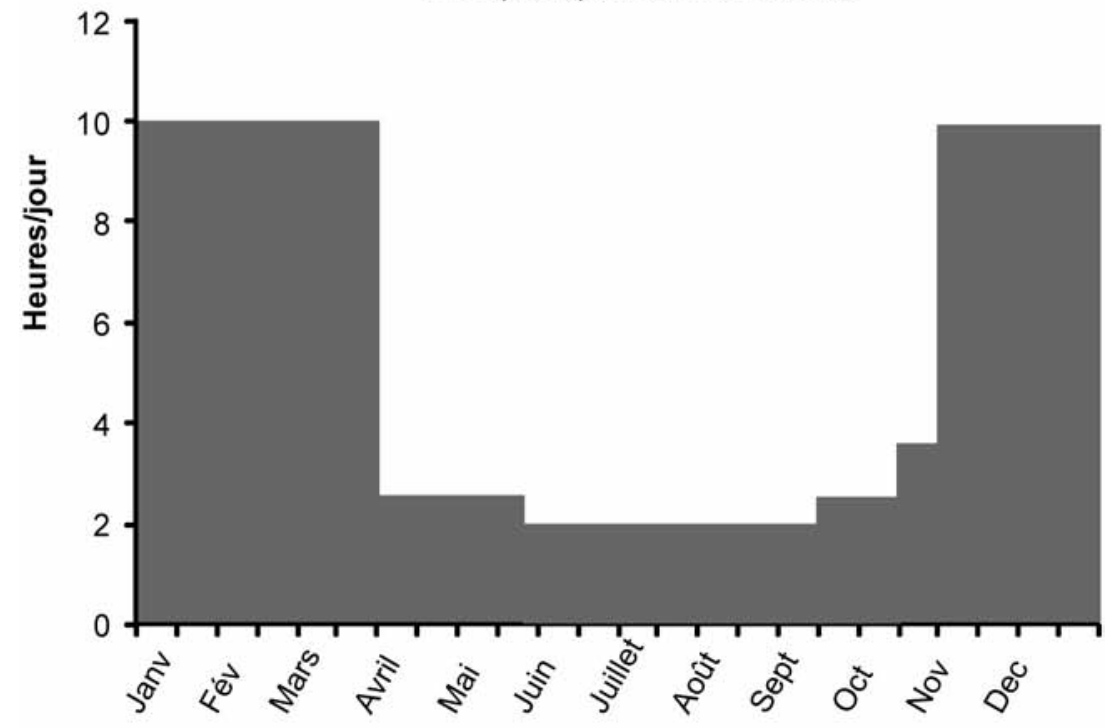

Profil en $U$ «rupture très marquée»

recherche sur des techniques d'élevage simplifiées, touchant le rationnement, le rythme de traite ou la configuration du système (par exemple la concentration des mises bas sur trois mois pour fermer la salle de traite). Les articles d'Agabriel et al (2012), de Hostiou et Fagon (2012), et de Martel et al (2012) dans ce numéro reviennent sur ce point.

b) Les choix de conduite et les raisons de travailler sont étroitement liés

Plusieurs auteurs comme Lémery (2003) et Commandeur (2006) ont mon- tré combien étaient associés les conceptions de métier (entrepreneur, artisan, animalier...) et les choix techniques des agriculteurs à travers les concepts de «farming styles» et d'identités professionnelles. Des choix techniques spécifiques peuvent également être porteurs du sens que les gens mettent dans leur travail (de l'identité, du relationnel, du goût pour la technique...), dans leur rapport aux animaux (Fiorelli et al 2007). Par exemple, la pratique du biberonnage, très consommatrice en temps, a d'autres significations pour les per- sonnes qu'une simple substitution de lait, car la dimension maternelle et l'attention à la vie jouent un rôle déterminant. De même, la suppression du gardiennage évoquée ci-dessus a des implications identitaires car ce métier est reconnu comme porteur de savoirfaire spécifiques (Moneyron 2003, Meuret 2010). Enfin, le choix d'une race, au-delà de toute évaluation zootechnique objective, atteste de l'appartenance à un groupe social.

c) Les volumes de travail jouent sur les modalités de pilotage du système d'élevage

Hémidy et al (1993) avaient souligné qu'une des voies de réduction de la complexité de gestion d'une entreprise consistait à définir des entités de gestion autonomes les unes des autres et les modalités de leur coordination. En élevage bovin allaitant, nos études ont mis en évidence que les formules d'allotement du troupeau et les successions de parcelles pâturées par les lots, sont sensibles aux pressions de travail exprimées $i)$ sur les critères de dimension (effectifs et surfaces), et par le nombre de travailleurs permanents (Ingrand et Dedieu 1996), et ii) par la dispersion du parcellaire (Josien et al 1994). L'existence de lots d'animaux autonomes dans leur composition toute l'année ou lors de la saison de pâturage montre l'existence de ces pressions de travail, alors même que l'application des recommandations alimentaires conduirait à régulièrement réalloter. Les circuits de pâturage expriment des arbitrages entre, d'une part la recherche d'une simplification de travaux jugés souvent comme impossibles à déléguer (manipulations, déplacements sur de longs trajets d'animaux, surveillance quotidienne) et, d'autre part l'ajustement de la diversité des ressources du territoire avec celles des besoins alimentaires des lots. Lorsque les pressions de travail s'accroissent, les circuits de pâturage tendent à se concentrer sur des «blocs» (ensemble de parcelles proches ou contigües pâturées par un seul lot pendant la saison de pâturage) indépendants les uns des autres (Dedieu et al 1997).

\section{d) Les attentes de travail influent sur} les procédures pratiques

Martel et al (2007) ont mis en évidence par enquête auprès d'éleveurs porcins, l'impact des attentes d'allègement du travail le week-end ou de maîtrise de la durée quotidienne du travail d'astreinte sur les procédures de gestion des mises bas (déclenchement ou non de la parturition, assistance, soins aux nouveaux nés) et des inséminations (durée et rythme de surveillance des chaleurs, 
Figure 3. Répartition des élevages porcins naisseurs sur les deux premiers facteurs de l'AFC portant sur les variables constituant le "planning des séances de travail» (Martel et al 2007).

Les deux premiers facteurs issus de l'AFC expliquent 23 et $17 \%$ de la variabilité issue des enquêtes. Ils regroupent pour le premier des variables liées à la charge de travail journalière (forte charge vs faible charge), et pour le deuxième des variables liées aux procédures des éleveurs lors des mises bas et pour la détection des œstrus le week-end.

Les aires numérotées correspondent aux classes identifiées par la classification ascendante hiérarchique.

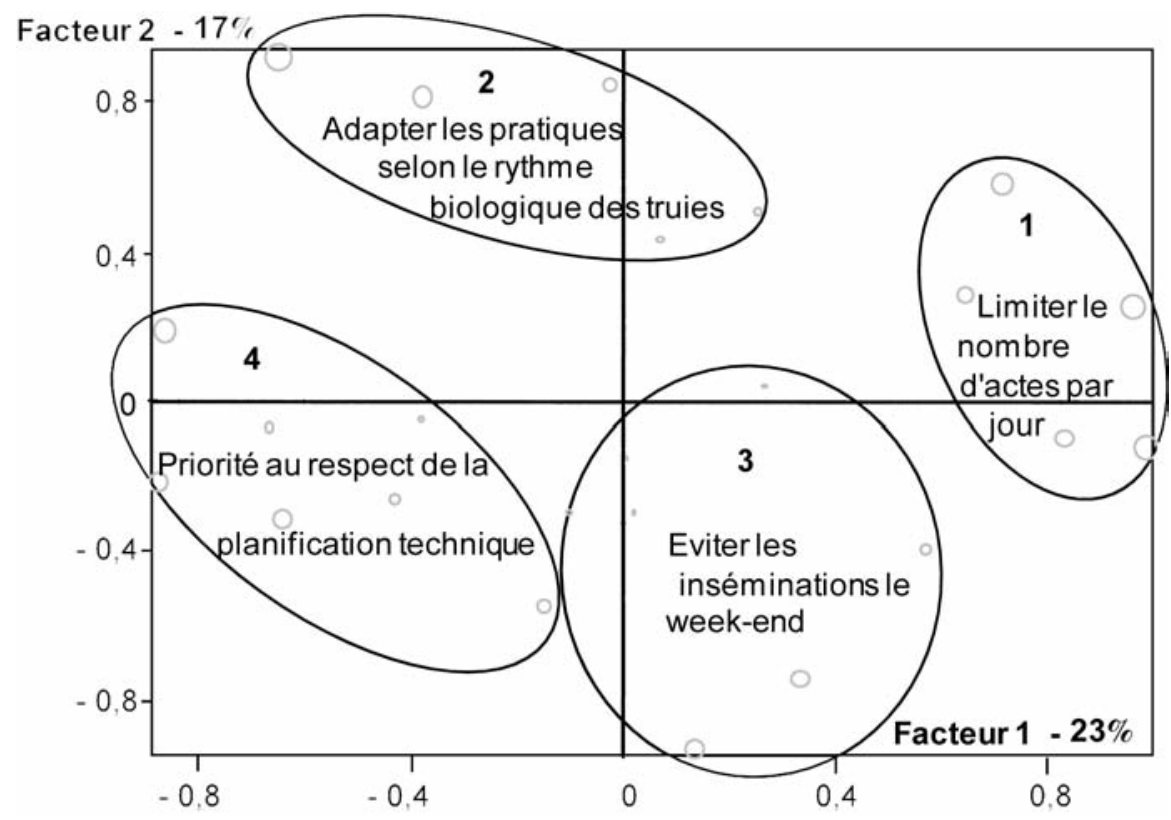

fréquences des inséminations artificielles successives). L'analyse, résumée à la figure 3 , conduit à distinguer quatre types d'attentes en matière de rythme de travail intra-hebdomadaire. Le premier type cherche à limiter le nombre des tâches de reproduction chaque jour, le second vise des pratiques de détection et de surveillance des mises bas calées sur le rythme biologique des truies, le troisième souhaite réduire le nombre de tâches de reproduction (notamment les inséminations) survenant le week-end. Le dernier type met en place des pratiques qui induisent de nombreuses sessions de travail par jour tout en évitant les mises bas survenant le week-end, il est dénommé «donner la priorité au respect de la planification technique». Ainsi, derrière l'apparente uniformité du modèle de production intégrée, nous retrouvons des procédures bien différenciées mettant en avant l'inclusion de préoccupations de travail des éleveurs au quotidien et ayant, le cas échéant, des implications sur les processus biotechniques en jeu (Martel et al 2008).

En conclusion, le constat général de diversité des systèmes d'élevage, y compris au sein d'une même filière de production indique que les éleveurs conservent une capacité d'adaptation forte de leurs conduites d'élevage selon leurs conditions propres et leurs attentes vis-à-vis de leur activité. Nos observations et celles, plus complètes, présentées par Hostiou et Fagon (2012) dans ce numéro, soulignent que la simplification des conduites est un levier d'ajustement entre la disponibilité en maind'œuvre et les besoins en travail. La simplification des procédures associées est un autre niveau d'ajustement, au plus près du travail réel tel que défini par les ergonomes au paragraphe 2.1. Les procédures qui mettent en contact l'éleveur et ses animaux (surveillance, contacts...) sont problématisées par l'éthologie animale (Boivin et al 2012) et la psychosociologie (Porcher 2011). Enfin, la liste des interactions que nous illustrons n'est pas exhaustive, le domaine de la santé, celui de la charge mentale et des compétences sont d'autres pistes de travail (ou de collaborations) à explorer dans l'avenir.

\section{3 / Qualifier l'organisation du travail}

Nous avons développé un cadre conceptuel spécifique de la zootechnie des systèmes d'élevage dans la mesure où il est explicite du point de vue de la conduite d'élevage, c'est-à-dire de la correspondance entre des pratiques, des durées, des rythmes de travail et des personnes (figure 4). Nous rappelons ici les apports de l'économie, de l'agronomie et de l'ergonomie à cette construction. Puis nous présentons le cadre conceptuel ainsi construit et les modèles opérationnels que nous avons mis au point.

\section{1 / Les emprunts aux discipli- nes}

Nous avons d'abord été intéressés par les contributions de l'économie à la mesure du travail agricole total. L'objectif était de rendre compte des évolutions de la durée du travail agricole dans les exploitations et de comparer les temps de travaux des agriculteurs avec d'autres catégories socioprofessionnelles (Brangeon et Jegouzo 1998). Il s'agissait de dépasser les limites des propositions classiques d'expression de la main-d'œuvre sous forme d'unités cumulées d'équivalent travailleur à temps complet (encadré 2) (Personne Année Travail (PAT), Unité de Main d'Euvre (UMO), Unité Travailleur Humain (UTH)) dont Lacroix et Mollard (1991) avaient souligné les grandes limites pour l'évaluation de la durée totale du travail agricole et l'analyse des critères économiques par unité de travail. Le chronométrage, adapté pour l'analyse de tâches élémentaires (par exemple pour déplacer une claie sur une distance donnée) (Iger 1977), ou d'opérations techniques bien délimitées comme la traite (Hémidy 1986), relevait des démarches d'organisation scientifique du travail. S'intéresser aux tâches élémentaires devait alors permettre des gains de productivité globale. Le chronométrage est inapplicable à l'échelle d'une exploitation, la variété des travaux, leur imbrication et leur caractère saisonnier étant trop important. Les méthodes de budget temps ou de budget travail, bâties sur un découpage des tâches selon des catégories simples (tâches d'élevage, de cultures, de gestion...), étaient également considérées comme peu fiables ou très sélectives des agriculteurs (beaucoup de défections, la méthode étant très lourde). La méthode de reconstitution analytique pour évaluer les temps de travaux annuels de Lacroix et Mollard (1991), croisait enquête déclarative et identification de points de repère temporels concrets pour les agriculteurs, au fur et à mesure du déroulement de la campagne annuelle. Elle a beaucoup inspiré les principes d'enquête de la méthode Bilan Travail (Dedieu 1993, Dedieu et al 2000).

L'ergonomie a été la deuxième discipline de référence dans la construction de notre cadre conceptuel. Les ergonomes ont découpé dans les bassins ovins laitiers les tâches selon les rythmes qu'elles imposaient aux travailleurs 
Figure 4. D'un calendrier technique, à un calendrier de travail sur lequel figurent les travaux et les travailleurs.

du calendrier technique ...

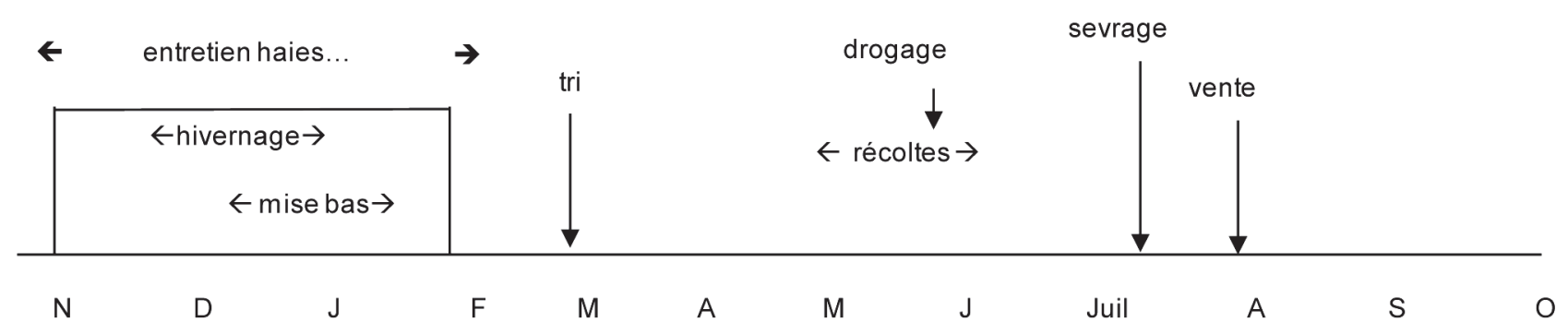

aux calendriers des travaux et des travailleurs
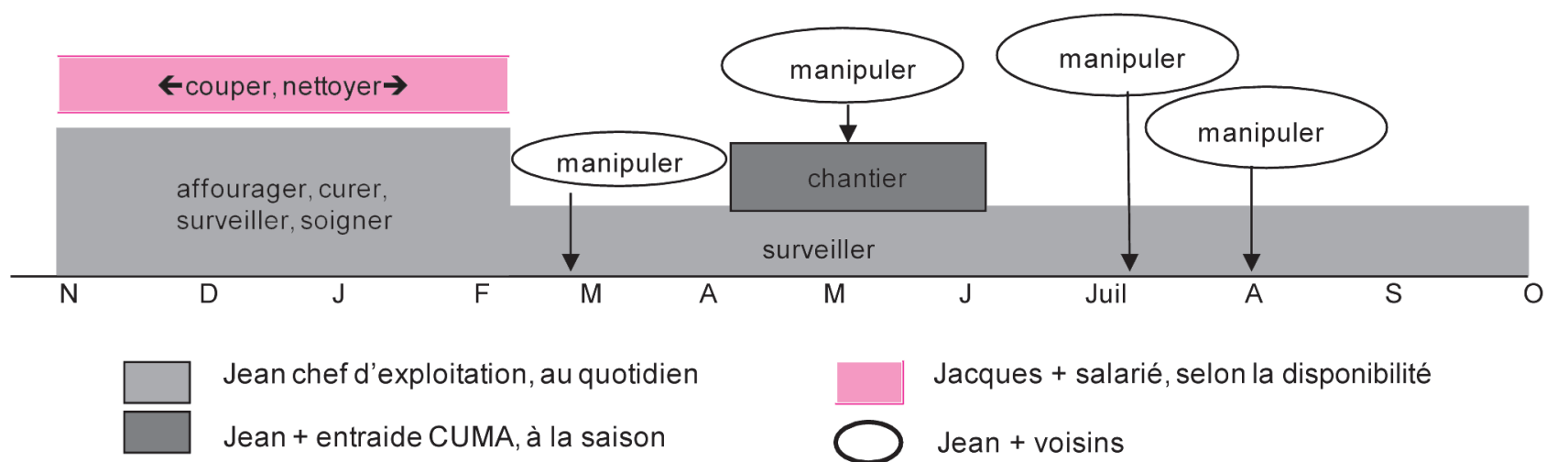

Jean chef d'exploitation, au quotidien

Jean + entraide CUMA, à la saison

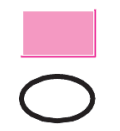

Jacques + salarié, selon la disponibilité

Jean + voisins (répétitif quotidien, saisonnier....) (Cellier et Marquié 1980, Bagès et Rieu-Gout 1981). Plus que les durées des travaux, l'articulation entre les tâches ayant des rythmes et des impératifs différents posait problème aux éleveurs. Valax (1989) s'intéressant au travail des femmes en agriculture avait décrit un travail fractionné, avec des butées temporelles incontournables (les repas, les enfants à accompagner...), qui rendaient problématique l'articulation des temps privés et professionnels.
L'agronomie dans son analyse des périodes de pointes du calendrier cultural, a proposé de rendre compte des concurrences entre travaux au travers d'une estimation des marges de manœuvre (Papy et al 1988). Il s'agissait moins de rechercher une combinaison optimale des moyens de production que d'envisager l'aptitude d'une organisation du travail donnée (incluant des ajustements possibles des itinéraires techniques et de la main-d'œuvre) à faire face à des années climatiques différentes.

Encadré 2. Les difficultés d'utilisation des équivalents travailleurs à temps complet.

La quantification statistique de la main-d'œuvre a fluctué.

- Ainsi en 1963, I'INSEE définissait la notion «Personne-Année-Travail» (PAT), c'est-à-dire un équivalent temps complet annuel, comme travaillant 9 heures par jour pendant 300 jours, donc 2700 heures par an. En 1967, la PAT correspondait à 8 heures par jour pendant 275 jours soit une forte réduction de la norme de travail en quatre années. En 1970, les personnes ont été classées en quatre catégories de temps (de moins d'un quart jusqu'à temps complet) et avec un coefficient selon le statut. Ainsi pour la plage «mi-temps à plein temps» un aide familial est comptabilisé pour 0,66 PAT, un chef d'exploitation pour 0,68 et, pour des raisons inconnues, un salarié à 0,78. En 1992 un temps complet est défini par une durée «au moins égale à 39 heures par semaine, ou à 20 jours par mois».

- En 2011, I'Unité de Travail Annuel (UTA) équivaut au travail d'une personne travaillant à temps plein pendant une année. Désormais aucune référence à une durée horaire connotée avec l'évolution de la législation sur le travail salarié ne figure dans la définition statistique. L'UTA définie par I'INSEE ne distingue pas le travail familial rémunéré de celui sans contrepartie financière. Elle ignore les travaux réalisés en entraide, c'est-à-dire avec une contrepartie en temps ou en prêt de matériel.

- Dans le cadre du dispositif Réseau d'Elevage (Institut de l'Elevage et Chambres d'Agriculture) les unités de main-d'œuvre bénévoles sont spécifiées et les calculs économiques concernent uniquement la main-d'œuvre rémunérée (UMOr), salariée ou non. Les traitements de la base de données permettent maintenant de calculer la main-d'œuvre imputable à chaque atelier herbivore (Charroin et al 2012).

Mesure du travail en tenant compte des évolutions saisonnières telles qu'elles se présentent au cas par cas, nécessité de rendre compte des rythmes différents du travail en élevage, estimation de la marge de manœuvre comme synthèse d'une approche de l'organisation, les bases du cadre conceptuel des zootechniciens étaient en germe dans ces différentes approches. Elles servent à rendre compte de ce qui marque, d'une exploitation à l'autre, des choix de conduite différents.

\section{2 / Le cadre conceptuel des zootechniciens}

Quatre propositions caractérisent ce cadre conceptuel.

\section{a) Des travaux différents}

Les travaux réalisés dans l'exploitation ne sont pas équivalents ni additifs, ils doivent être distingués selon leur aptitude à être différé et leur rythme. Le travail quotidien répétitif est assez emblématique de l'élevage et son articulation avec d'autres travaux ayant des caractéristiques temporelles variées est la principale difficulté de l'organisation du travail. Avec l'entrée par l'aptitude à être différé d'une journée à l'autre, nous distinguons le travail d'astreinte (qui ne peut pas être différé) et le travail de saison (qui peut être différé). Avec l'entrée par le rythme, le travail d'astreinte peut être quotidien (soins aux animaux, 
traites qui se répètent d'un jour à l'autre), ou non quotidien (comme la vente en marché qui a lieu à date fixe dans la semaine). En rendant compte de ces différences de caractéristiques temporelles des tâches, nous renonçons à une expression univoque des tâches (de leur durée notamment), mais nous qualifions le lien entre des pratiques (domaine zootechnique) et les tâches (domaine du travail) selon ces catégorisations.

\section{b) Des travailleurs différents}

Tous les travailleurs ne sont pas équivalents de par leur fonction dans le collectif, leur rythme de présence (Allaire 1988) et le type de contrepartie à leur participation au travail. La cellule de base comprend les travailleurs permanents pour qui l'activité agricole est prépondérante en temps et en revenu (l'exploitant, le couple d'exploitants, les associés). Elle correspond aussi au noyau organisateur du travail agricole. L'organisation rend compte spécifiquement de leurs attentes en termes de qualité, rythme, efficacité du travail et de la nécessité de composer avec les impératifs liés à leurs éventuelles autres activités. La main-d'œuvre hors cellule de base comprend les bénévoles, l'entraide (contrepartie en jours de travail rendu), l'entreprise et le salariat (contrepartie en argent). Toutes ces personnes n'ont pas la même disponibilité en temps, certains travailleurs sont permanents (par exemple les retraités), d'autres sont temporaires, ou interviennent de façon ponctuelle. Elles n'ont pas les mêmes compétences, peuvent être polyvalentes ou spécialisées sur certaines tâches.

Cette catégorisation ne se substitue pas aux notions d'UTA et d'UMO des économistes, car elle se situe sur un autre plan : celui de l'organisation. Il s'agit de rendre compte des modalités d'organisation de la main-d'œuvre (répartition du travail entre les personnes, délégation, partage) selon les types de travaux (travail d'astreinte et travail de saison). Ainsi peuvent émerger les dimensions familiales (bénévolat du frère, plutôt sur le travail d'astreinte), collectives (entraide, CUMA, plutôt sur le travail de saison) et d'externalisation (entreprise, salariat temporaire ou permanent, service de remplacement) de cette organisation.

\section{c) Des échelles temporelles variées}

L'organisation du travail à l'échelle de l'année résulte de l'enchaînement de périodes aux caractéristiques organisationnelles différentes, notamment par l'évolution des tâches agricoles à réaliser (des travaux à l'extérieur et des travaux à l'intérieur des bâtiments par exemple). Ces périodes marquent une stabilité du travail d'astreinte quotidien (du contenu des tâches et des travailleurs qui en ont la charge), voire de sa durée et des modalités d'interaction avec les autres tâches. Ces périodes ne sont pas définies a priori mais expriment bien des modalités d'interaction spécifiques à chaque cas entre les impératifs techniques, les rythmes de présence des travailleurs, le poids des attentes et l'importance des autres activités de la cellule de base sur l'organisation du travail.

\section{d) Evaluation de l'organisation $d u$ travail}

L'organisation du travail peut être qualifiée et évaluée à partir d'indicateurs quantitatifs et qualitatifs aux différentes échelles de temps (période, année) évoquées ci-dessus (Madelrieux et Dedieu 2008). Des variables quantitatives rendent compte des dimensions (cheptel, surfaces), des temps de travaux d'astreinte et de saison, et de la part réalisée par la cellule de base (degré d'autonomie dans la réalisation du travail). Des variables qualitatives rendent compte $i$ ) de la composition de la main-d'œuvre, ii) des modalités d'agencement entre les différentes personnes et les différentes catégories de travail (qui fait quoi; en autonomie, en partage, en délégation), iii) de la conduite d'élevage (troupeau, ressources), des équipements et bâtiments, des autres activités.

L'évaluation de l'organisation du travail porte sur les deux dimensions que sont l'efficience et la flexibilité. L'efficience du travail est estimée à partir d'indicateurs comme le temps de travail d'astreinte par unité productrice (UGB, vache, brebis...), ou produite (1000 L de lait par exemple). Cette dimension, très importante car la compétitivité est une exigence forte, s'appuie largement, hors production de niche, sur la productivité du travail. $L a$ flexibilité de l'organisation (Dedieu et al 2008b) est approchée par l'estimation des marges de manœuvre en temps des agriculteurs pour réaliser les tâches non comptabilisées et les activités privées, une fois réalisées leurs parts de travail d'astreinte et de saison. L'évaluation se fait au travers de l'indicateur Temps Disponible Calculé (TDC) (Dedieu et al 2000). La flexibilité renvoie également à la capacité de l'organisation quotidienne du travail à faire face à des fluctuations du travail liées à des changements de main-d'œuvre (par exemple les jours avec ou sans le salarié du groupement d'employeurs), des concurrences fortes entre travaux (jour de marché), ou des aléas climatiques (jour de pluie ou de beau temps). L'analyse porte alors sur l'analyse du nombre de jour- nées types (en termes de répartition du travail quotidien entre les personnes) et des modifications apportées à ces formes d'organisation au cours de l'année (Madelrieux et al 2009, Hostiou et Dedieu 2011).

\section{4 / Les productions de connaissances et de métho- des sur le travail et son organisation}

Nous résumons dans cette partie quelques-unes des productions issues du cadre conceptuel présenté précédemment : des modèles du travail, des connaissances portant sur les facteurs de variation des temps de travaux et sur les formes d'organisation du travail.

Les modèles du travail produits par les zootechniciens sont résumés dans l'encadré 3. Ils sont utilisés tant par la recherche que par le développement pour produire des connaissances sur la diversité des situations en fermes, des référentiels de temps de travaux ou comme cadre pour le développement d'outils de conseil et de formation. Nous reviendrons plus en détail sur ces points dans la partie suivante.

Les nombreuses études réalisées sur les facteurs de variation du temps de travail d'astreinte, mettent en avant un effet de la taille du cheptel en interaction avec d'autres facteurs tels que les choix de conduite (durée d'hivernage, nombre de périodes de mises bas et leur étalement), les travailleurs (les retraités ne comptent pas leur temps...), les bâtiments et équipements. Ces interactions complexes impliquent une très grande variabilité des valeurs d'efficience (des écarts de 1 à 4) y compris pour des classes de taille de cheptel identiques (figure 5). Pour le travail de saison, l'effet taille est prédominant ainsi que l'assolement et l'utilisation des fourrages (part des cultures, intensification des surfaces en herbe) (encadré 4).

Les marges de manœuvre en travail des exploitants sont également très variables (de 0 à 1800 heures de TDC par an). Les analyses soulignent que les marges de manœuvre élevées (1200 heures et plus par an et par personne) s'expliquent par des stratégies explicites d'organisation s'appuyant sur la simplification des conduites, l'automatisation, l'accroissement de la puissance des machines et la délégation du travail. Les investissements lourds dans des bâtiments et équipements de pointe peuvent constituer en soi une stratégie visant à dégager de fortes marges de manœuvre. La simplification des 
Encadré 3. Les modèles du travail développés par les zootechniciens.

La méthode Bilan Travail (Dedieu et al 2000). Par reconstitution analytique telle que proposée par des économistes (Lacroix et Mollard 1991) le Bilan Travail quantifie le travail lié à la conduite des troupeaux et des surfaces (tous travailleurs concernés) et évalue la marge de manœuvre des exploitants pour la réalisation d'autres activités sur l'exploitation et disposer de temps libre. Plus de 4000 enquêtes ont été réalisées en France. La méthode sert de support à plusieurs outils de conseil comme «Travibov» - conseil en élevage bovin viande (Sarzeaud et Chauvat 2010). Elle est mise en œuvre au Brésil (Hostiou et Dedieu 2009), en Uruguay (Correa et al 2011) et au Vietnam (Hostiou et al 2010).

La modélisation Atelage (pour "activités de travail dans les exploitations d'élevage») (Madelrieux et al 2006, 2009) s'inspire du concept de système d'activités des ergonomes (Curie et Hajar 1987). Atelage propose une démarche de qualification de l'organisation du travail à partir de l'expression des différentes formes du «qui fait quoi» et des liens entre activités d'astreinte et activités de saison (subordination, juxtaposition...) au cours d'une campagne agricole. Cette organisation intègre la façon dont interagissent la conduite d'élevage, la main-d'œuvre, les autres activités (agricoles, non agricoles, privées). L'organisation du travail est envisagée comme un système d'activités de travail en interaction dynamique, intégrant les régulations liées aux aléas climatiques et de disponibilité des personnes. L'outil Cote (Changements et organisation du travail en exploitation d'élevage) constitue un exemple de valorisation du modèle dans le champ des outils du conseil (Calland et al 2009).

Le modèle Quaework (Hostiou et Dedieu 2011) s'inspire des deux approches précédentes. II vise à qualifier l'organisation du travail et à en évaluer l'efficience et la flexibilité à partir des données de temps de travaux, de marge de manœuvre et de régulations (caractéristiques et nombre de journées-type). II est en cours de test pour l'analyse de systèmes d'élevage innovants (exploitations unipersonnelles, grandes fermes avec salariés...).

Figure 5. Dispersion de l'efficience du Travail d'Astreinte (TA/vache laitière) de l'atelier bovin laitier (Fagon et Sabatté 2010).

La variabilité pour une même classe de cheptel s'explique par des différences d'équipement (robot, stabulation libre/logettes...), de zone de production (plaine ou montagne), de structures d'exploitation et de complexité de systèmes (spécialisation vs mixité).

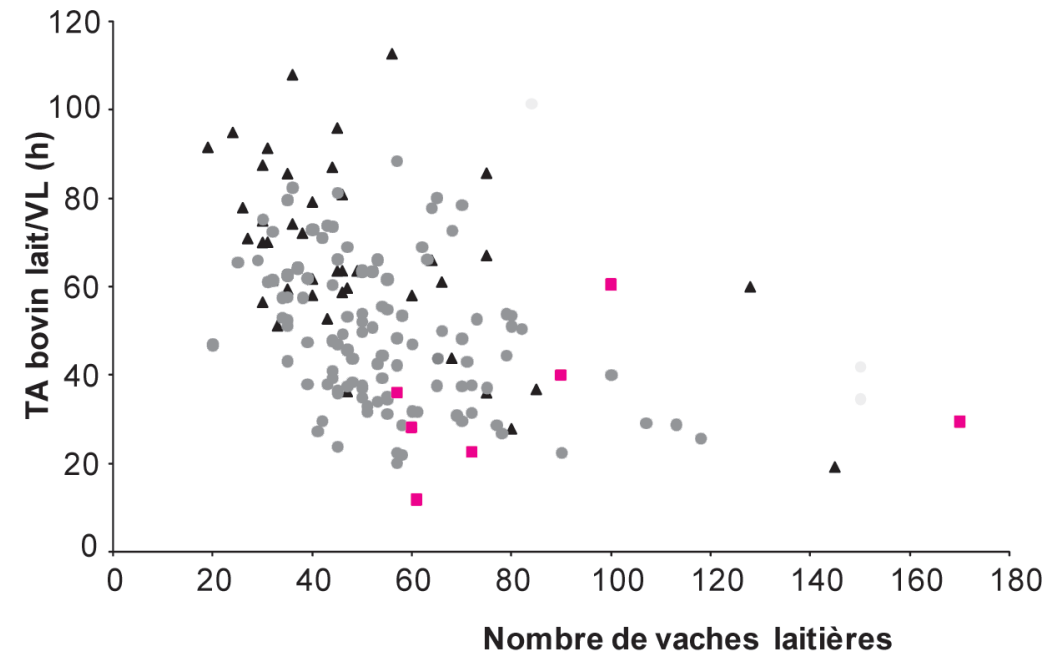

$\Delta$ Montagne - Plaine Exploitations avec robot de traite ou rotolactor

Encadré 4. Facteurs de variation de l'efficience du travail de saison par hectare.

L'effet d'économie d'échelle de la taille de la Surface Fourragère Principale (SFP) sur le travail de saison par hectare s'explique principalement par l'opportunité d'utiliser des matériels plus performants. Ainsi le traitement de 137 Bilans Travail en ovin allaitant montre que pour trois classes de SFP (moins de 45 ha, de 45 à 75 ha, plus de 75 ha) les temps de travail de saison sur la SFP par ha de SFP, sont respectivement de 1,6 j, $1 \mathrm{j}$ et 0,6 $\mathrm{j}$ (Chauvat 2010).

Ce critère est également dépendant du degré d'intensification, ainsi il passe de $0,8 \mathrm{j} / \mathrm{ha}$ SFP en moyenne pour des exploitations bovines laitières de plaine «herbagères» récoltant $2,5 \mathrm{t}$ de fourrage par UGB $(n=25)$ à 1,2 j pour celles «avec beaucoup de maïs dans la SFP» et récoltant 4 t de MS par UGB ( $n=58)$ (Fagon et Sabatté 2010).

L'importance des répartitions de temps par opération culturale (implantation, fertilisation, traitements, entretien, récolte) est illustrée en élevage ovins de Poitou-Charentes. Pour les élevages spécialisés $(n=19)$ qui souvent pâturent l'hiver, le temps passé à l'entretien des surfaces, notamment au gyrobroyage, est nettement plus élevé ( 9 j pour 86 ha de SFP) que dans les élevages ovins-bovins ( 4 j pour $105 \mathrm{ha}$ ), et a contrario plus faible pour la récolte de fourrage (20 vs $36 \mathrm{j}$ ) (Bellet 1999). conduites d'élevage est souvent associée à une délégation forte d'une partie du travail. Le tableau 1, comparant deux situations d'élevage ovin montre comment les différences de temps de travail d'astreinte résultant des choix techniques ainsi que la sous-traitance du travail de saison aboutissent à des marges de manœuvre en temps pour les éleveurs allant du simple au double.

Les données sur la flexibilité des formes d'organisation quotidienne du travail indiquent plusieurs possibilités d'adaptations lorsque le contenu et la durée du travail changent. Dans certaines situations, la répartition du travail entre les personnes reste identique, tandis que dans d'autres elle dépend de la nature de la modification (travail de saison tel un chantier de récolte $v s$ travail d'astreinte lors de la présence du salarié du groupement d'employeurs). Ces modèles des zootechniciens ne peuvent éclairer l'ensemble des dimensions du travail évoquées dans la première partie, mais constituent des éléments d'une réflexion globale. L'organisation est alors reliée aux facteurs sociologiques jouant sur l'importance de la délégation (Pichereau et al 2004) ou aux représentations du travail que portent les éleveurs (Cittadini et al 2001, Dufour et Dedieu 2010). Fiorelli (2010) a ainsi proposé un cadre d'analyse interdisciplinaire des conditions de vie au travail intégrant quatre volets : les rationalités subjectives, les caractéristiques du système d'activités du ménage (activités économiques et privées), les contraintes temporelles entre activités (calendaires, quotidiennes) et l'organisation du travail (figure 6). Ce cadre permet de mettre à jour les tensions qui s'exercent entre ce qu'attendent les personnes de leur travail en élevage et ce que produit l'organisation, et de repérer ce qui est central dans l'association entre ces deux dimensions. Certaines situations de malaises d'éleveurs ou de salariés s'expliquent ainsi par le décalage entre les impératifs d'efficience et celles d'une relation paisible avec les animaux dans les grands troupeaux (Fiorelli et al 2012).

\section{5 / Le travail en élevage : un processus partenarial}

Ces vingt années sont aussi celles de la création ex nihilo d'une véritable communauté de recherche, formation et développement sur ce thème, nourrie d'une coopération permanente avec comme institutions pivots, l'INRA (département SAD) et l'Institut de l'Elevage (Département Actions Régionales). Elle a pris différentes formes, longtemps informelles, et plus 
Tableau 1. Marges de manœuvre en temps évaluées par le Temps Disponible Calculé dans deux exploitations ovines herbagères. Les deux élevages ont des structures voisines (800 brebis, 1 travailleur permanent, 140 ha). Le Bilan Travail permet d'évaluer l'organisation du travail sur l'exploitation à l'échelle de la campagne. Les différences de temps de travail, d'astreinte et de saison, ainsi que la part de sous-traitance aboutissent par construction à des résultats de marges de manœuvre en temps pour les éleveurs allant du simple au double.

\begin{tabular}{|c|c|c|c|c|}
\hline Exploitations & A & Com mentaires & B & Commentaires \\
\hline $\begin{array}{c}\text { Travail d'astreinte (h/an) } \\
\text { fait par léleveur }\end{array}$ & $\begin{array}{l}1664 \mathrm{~h} \\
100 \%\end{array}$ & $\begin{array}{c}\text { Conduite du troupeau } \\
\text { simplifiée }\end{array}$ & $\begin{array}{l}2312 \mathrm{~h} \\
90 \%\end{array}$ & $\begin{array}{c}\text { Conduite du troupeau } \\
\text { exigeante et bâtiments peu } \\
\text { adaptés }\end{array}$ \\
\hline $\begin{array}{l}\text { Travail de saison (j/an) } \\
\text { fait par léleveur }\end{array}$ & $\begin{array}{l}110 j \\
52 \%\end{array}$ & Délégation des travaux & $\begin{array}{l}95 \mathrm{j} \\
97 \%\end{array}$ & $\begin{array}{l}\text { Matériel performant, } \\
\text { autonomie de realisation }\end{array}$ \\
\hline $\begin{array}{c}\text { Temps Disponible } \\
\text { Calculé pour l'éleveur } \\
\text { (h/an) }\end{array}$ & $1000 \mathrm{~h}$ & $\begin{array}{l}\text { Marge de manceuvre } \\
\text { en temps satisfaisante }\end{array}$ & $500 \mathrm{~h}$ & Situation tendue \\
\hline
\end{tabular}

récemment organisées dans le cadre du Réseau Mixte Technologique (RMT) «Travail en élevage». Ce RMT regroupe différents acteurs du développement (Chambres, Instituts, CUMA), de l'enseignement (supérieur et technique) et de la recherche (INRA, Irstea, CIRAD) autour de productions scientifiques, d'outils de conseil et de documents pédagogiques, de rencontres, d'actions innovantes (encadré 5). Ce processus partenarial s'est nourri de la consolidation progressive du cadre conceptuel zootechnique que nous avons présenté ci-dessus, de l'ouverture vers les sciences sociales (sociologie du travail, diagnostic ergonomique de 1'activité), du développement d'outils de conseil.

Figure 6. Cadre d'analyse de l'aménagement des conditions de vie au travail (Fiorelli 2010).

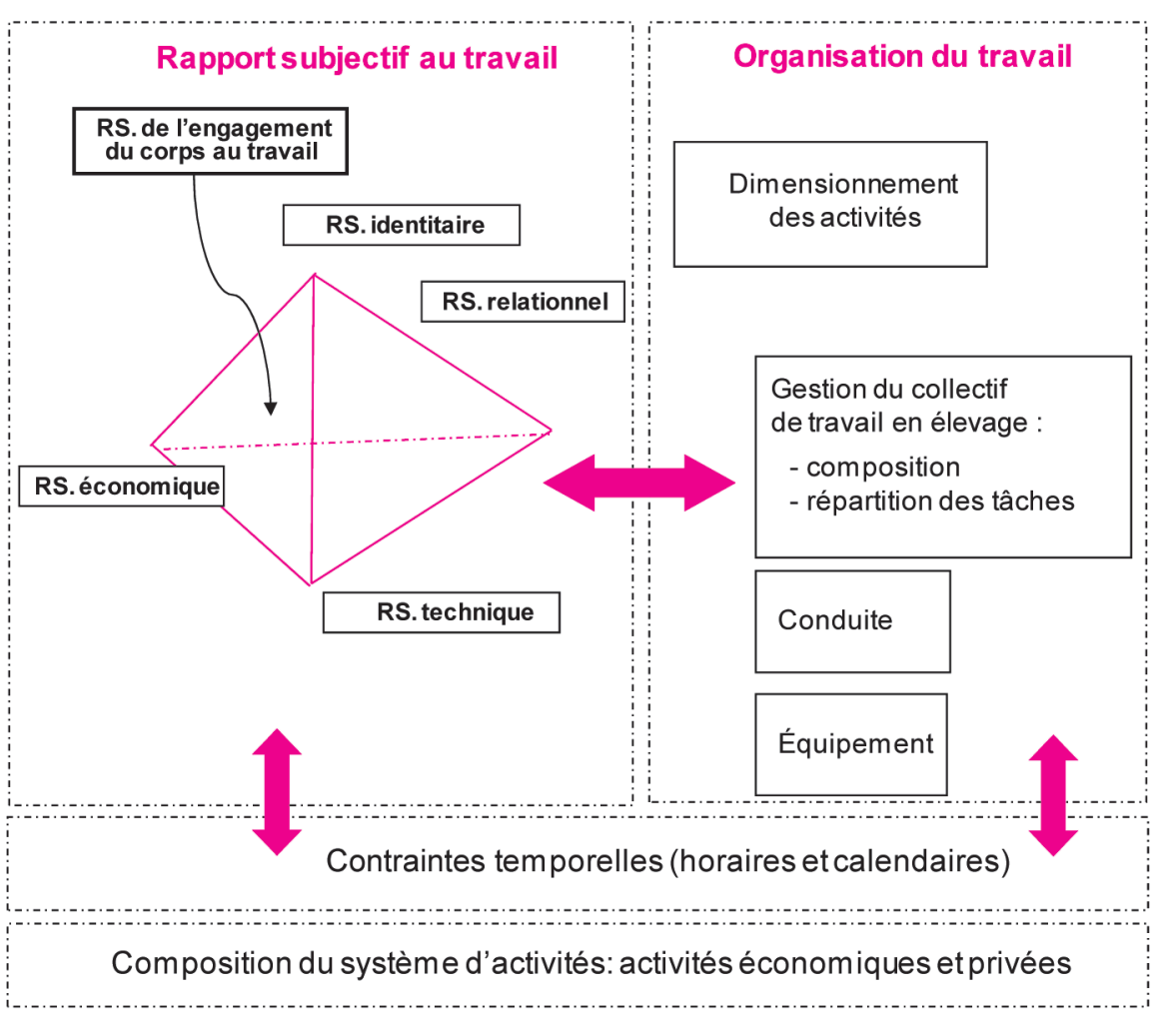

retraités) et que celles-ci ne pouvaient être ignorées alors même que nous prétendions développer une approche systémique de l'élevage. Cette coopération a aussi calibré très fortement les initiatives et les produits qui devaient convenir aux deux parties recherche et développement. Le Bilan Travail a ainsi été configuré explicitement de façon à être compatible avec les charges et us de travail des techniciens, avec une enquête d'une demi-journée plutôt que des enregistrements ou des budgets temps.

Différentes phases, scandées par les trois Rencontres Nationales des acteurs du Développement, de la Recherche et de la Formation à Clermont-Ferrand (1996), Poitiers (2004) et Rennes (2009), ont permis l'élargissement progressif de la communauté.

Nous pouvons résumer les produits $\mathrm{du}$ partenariat selon 5 rubriques.

\section{1 / Des référentiels de temps de travaux par grand type de systè- me de production}

Le Bilan Travail visait à compléter la démarche d'analyse systémique du fonctionnement des exploitations d'élevage dont les Réseaux d'élevage étaient un des porteurs principaux. Ainsi, la méthode a été conçue pour que les techniciens et ingénieurs «références» des Chambres d'Agriculture puissent l'intégrer, à côté du bilan économique, du bilan fourrager et du bilan de reproduction, dans leurs boîtes à outils techniques. Afin de faciliter les calculs et la centralisation des données, un logiciel éponyme a été créé à la fin des années 1990. C'est aussi sur le dispositif national «Réseaux d'élevage» que s'est appuyée la formation des agents de développement à l'approche du travail par des zootechniciens et à sa déclinaison opérationnelle dans la méthode Bilan Travail. La multiplication des enquêtes a permis une première analyse quantitative sur des échantillons 
nationaux en 1996 (Jordan et al 1996) et la construction de référentiels inclus dans les modélisations de systèmes publiées sous forme de «cas-type». Avec la création du RMT Travail et la prise en compte des élevages granivores, sept filières animales (bovins lait et viande, ovins lait et viande, caprins, porcs et volailles) sont maintenant concernées par des références sur les temps de travaux (Cournut et Chauvat 2012).

\section{2 / Des références au conseil sur le travail}

Les groupes de développement, qu'ils soient institutionnels (syndicalisme, Réseaux d'élevage, Chambres d'Agriculture...) ou occasionnels (sessions de formation d'agriculteurs) ont voulu très rapidement après les premières analyses de groupes, «passer des références au conseil». D'une expression individuelle de problèmes de travail, les groupes évoluent vers une discussion collective structurée autour des données d'analyse $\mathrm{du}$ travail et de son organisation. Souvent ces réunions sont suivies de visites de fermes à tour de rôle pour débattre de l'organisation du travail. Les agriculteurs expriment leurs avis, voire leurs critiques, sur les pratiques de travail réel de leur collègue et envisagent ensemble des solutions. La réflexion peut s'engager aussi sur l'embauche à plusieurs d'un salarié (groupements d'employeurs) ou sur l'opportunité de modifier les choix de conduite (passage à la monotraite). Dans l'ensemble de ces dispositifs, que ce soit en groupe ou lors d'un conseil individuel, le conseiller est moins un prescripteur de solutions qu'un accompagnateur d'une réflexion sur un sujet où les points de vue personnels de chacun (sur le travail avec les autres, les accords familiaux...) sont en jeu et s'expriment, au-delà de l'apparente objectivité des durées de travail et des descripteurs de l'organisation. Ce travail d'accompagnement s'est appuyé sur le développement de nombreux outils de diagnostic et de conseil conçus à l'échelle régionale ou par filière, lesquels s'inspirent de l'un ou de l'autre des modèles du travail évoqués dans l'encadré 3, avec une attention plus ou moins grande accordée aux dimensions sociologiques et psychologiques du travail.

De fait, les formes et finalités des opérations de développement sont très dépendantes des volontés professionnelles et du profil des intervenants (conseillers d'entreprise, techniciens de filières, d'organisations de producteurs). Les résultats et pistes d'amélioration (le plus souvent illustrées par des témoignages d'éleveurs) sont présentés également lors de journées portes ouvertes en fermes et en stations expérimentales, de «rallyes travail» et à l'occasion de salons professionnels. Chauvat et Sabatté (2009) ont recensé vingt-neuf expériences de développement et de conseil regroupées en trois rubriques (dispositifs de conseil pour les éleveurs, formations des conseillers et des éleveurs, outils d'accompagnement) indicatrices de la variété des finalités des interventions. Les sujets évoqués peuvent concerner la globalité de l'exploitation ou un atelier, un thème particulier (le travail en bâtiments, les relations humaines) ou une période de la vie de l'exploitation (par exemple l'installation).

\section{3 / Du conseil au dispositif d'appui aux conseillers}

L'évaluation des actions de conseil sur le travail réalisée en 2009 montre que les conseillers s'approprient sans difficulté, voire sans formation spécifique, les outils proposés et souvent les adaptent à leur situation professionnelle (Kling et al 2012). Ils construisent un équilibre qui leur est propre entre les données quantitatives qui les sécurisent et des approches plus qualitatives indispensables à une relation de conseil individualisée. Ils sont très demandeurs d'un dispositif de coordination régionale des actions et des intervenants, qui requiert une volonté politique professionnelle et des moyens financiers (dit autrement «un moteur et du carburant»).

Même si la question initiale porte sur l'efficience du travail et la compétitivité de l'entreprise, l'articulation entre un projet de vie et un projet professionnel est le plus souvent au cœur du débat de l'accompagnement. Le conseiller doit alors trouver une juste distance entre d'une part, les compétences techniques, les référentiels de temps de travaux, la caractérisation des formes d'organisation et d'autre part, l'écoute des aspirations individuelles, des raisons de travailler en élevage (Kling et al 2012).

\section{4 / La formation}

Grâce au partenariat avec des professeurs de zootechnie ou plus souvent de gestion dans les lycées agricoles, les chargés de suivi des Réseaux d'élevage ont valorisé, dans de nombreuses régions, leurs analyses de groupe auprès d'élèves. Les expériences avec des apprentis en CFPPA, des étudiants en BTS, licence professionnelle ou écoles d'ingénieurs, à l'occasion de cours, de rapports de stage ou de tests de méthodes (de travail, d'analyse de données) ont été nombreuses. Elles montrent que le stage en exploitation agricole est une bonne occasion d'analyser le travail, de comparer les résultats avec les référentiels et de discuter avec les maîtres de stage du métier et du style de vie qu'implique l'élevage. Les exploitations de lycée agricole sont aussi des supports pédagogiques pour des projets d'élèves, comme par exemple l'étude des conséquences de l'installation d'un robot de traite sur le travail des salariés (Vincent et al 2011).

Ces opérations ponctuelles ont débouché sur une formation de formateurs de l'enseignement agricole, suivie de la publication d'un ouvrage pédagogique réalisé avec le concours de Montpellier Supagro antenne de Florac (Bishoff et al 2008). A l'occasion de la réforme du Brevet Professionnel option Responsable d'Exploitation Agricole (BPREA), une nouvelle unité capitalisable «Gérer le travail dans l'exploitation agricole» a été discutée avec les porteurs du RMT et validée par l'Inspection de l'enseignement agricole. Le thème de travail est bien plus explicite dans les parcours de formation qu'il ne l'était par le passé.

Encadré 5. Le Réseau Mixte Technologique Travail en élevage animé par l'Institut de l'Élevage, l'Inra et les Chambres d'Agriculture.

Ce RMT, labellisé par le Ministère de l'Agriculture en 2007, vise à amplifier les synergies entre filières, entre disciplines, entre territoires ainsi qu'à favoriser la constitution de pôles d'expertise pour les professionnels de l'élevage et les pouvoirs publics. II propose et coordonne des actions de conseil, de recherche, de formation et les met en perspective par des collaborations internationales. II s'appuie sur le site web de l'Institut de l'Elevage et diffuse régulièrement «La lettre d'information du RMT Travail» à plus de 600 personnes.

Les Rencontres nationales des acteurs du développement, de la recherche et de la formation (Rennes en 2009) comme les nombreuses publications (référentiels et outils de conseil), les participations aux salons professionnels et à des congrès scientifiques (français et européens) sont marquées par la diversité des partenaires du réseau.

\section{5 / Une ouverture internatio- nale}

La communauté recherche, formation et développement a développé surtout des relations avec des structures équivalentes dans les pays du Sud qui partagent nos interrogations sur le devenir de l'exploitation familiale. Au-delà des différences structurelles et d'équipements, l'analyse comparative d'exploitations d'élevage dans plusieurs pays (France, Vietnam, Uruguay, Argentine et Brésil) 
indique que la diversité des formes d'organisation du travail s'appuie sur les mêmes axes discriminants (Cournut et al 2010) (encadré 6). Une réflexion transversale est possible. Nos approches contribuent à débattre de la nature du travail familial et explorent pourquoi il est souvent présenté comme un frein à l'adoption d'innovations a priori favorables à la durabilité (Hervé et al 2002, Muchagata et Brown 2003). Hostiou et Dedieu (2009) ont ainsi montré en Amazonie que les prescriptions techniques d'intensification fourragère, exigeantes en travail d'astreinte, n'étaient adoptées que dans des exploitations spécialisées bien pourvues en main-d'œuvre permanente.

Dans les pays de l'OCDE, de moins en moins de jeunes sont intéressés par le métier d'agriculteur ou de salarié d'exploitation, comme si les rythmes, la durée du travail et plus largement les conditions de vie au travail étaient en train de tuer une profession devenue marginale qui n'hésite pas à dire "tant de travail pour si peu de revenu» (au moment de la crise du lait), ou «travailler plus pour gagner moins, non merci» (Ouest France, 23 mai 2011). Le site web d' 'AAustralian Dairy» est à ce titre tout à fait explicite : "The People in Dairy program is designed to assist dairy farmers with building the people part of their business [Le programme People in Dairy est conçu pour accompagner les éleveurs en donnant une consistance à la dimension humaine et collective de leur entreprise]». Ainsi, le sens du métier et le travail des personnes qui sont au cœur des réflexions pour l'avenir du secteur dans ces pays (Crawford et al 2010) peuvent être partagés avec notre communauté.

\section{6/Conclusion - Perspectives}

Le thème du travail en élevage et même en agriculture, bien que très présent dans les discours professionnels ou scientifiques à des titres divers, n'a jamais été porteur de programmes incitatifs de recherche et développement, lesquels ont été centrés tour à tour sur l'extensification, le développement des signes de qualité, la durabilité. Il s'en suit que les recherches sur le travail et la capacité à mobiliser le développement ont été adossées le plus souvent à d'autres thématiques. Ce n'est que récemment $i$ ) qu'un conseil spécifique sur le travail émerge, avec ses besoins de référentiels, de solutions techniques, et avec un changement de posture dans l'accompagnement des projets des éleveurs, ii) que les réflexions prospectives sur l'élevage (Peyraud et Baumont 2010) intègrent explicitement un volet sur les évolutions des structures et du travail des éleveurs, iii) que les acquis dans ce secteur sont considérés comme utiles pour aborder un ensemble plus vaste, au-delà de l'agricole, celui des très petites entreprises.

Des recherches et connaissances produites avec les éleveurs et les organismes de recherche et développement et de conseil, résultent deux acquis principaux. Le travail est essentiel pour l'analyse des systèmes d'élevage herbivore et granivore, la compréhension de leurs fonctionnements et de leurs transformations. Le travail pour un éleveur est à la fois un facteur de production économique, une succession de tâches et de chantiers à organiser et une activité qui construit l'identité professionnelle et personnelle de chacun, homme ou femme, exploitant ou salarié. Ces acquis impliquent pour les prochaines années de renforcer les synergies entre les disciplines et les métiers dans des déclinaisons variées (sciences techniques et sciences sociales, approches quantitatives et qualitatives, globales et par atelier) en ouvrant sur des domaines que nous n'avons fait qu'effleurer jusqu'à présent : la santé au travail, les relations humaines, la dynamique des compétences et des métiers. L'avenir requiert également de renforcer les interactions avec les disciplines constitutives de la zootechnie, notamment la génétique, la physiologie, l'éthologie et la santé animale. Les nouvelles technologies qui leur sont liées auront beaucoup d'impact sur le travail dans les exploitations moyennement à fortement capitalisées. Elles ouvrent également des perspectives quant aux opportunités de simplifications techniques s'appuyant sur une plus grande robustesse des animaux.

Les modèles techniques de l'élevage de demain qu'ils soient marqués «agroécologiques» ou «de précision» seront insérés dans des dynamiques d'agrandissement, et/ou de spécialisation, et/ou de diversification, et/ou de développement de niches commerciales. Les formes d'exercice de l'activité resteront diverses et controversées : les «megafarms» avec salariés sont présentées comme des perspectives incontournables ou a contrario comme inapplicables en France ; il en est de même des fermes unipersonnelles vues comme un renouveau de l'agriculture familiale ou, à l'opposé, un prélude à sa disparition. C'est l'ensemble de ces éléments de diversité qu'il faut envisager dans leurs interactions, ce qui implique de traiter conjointement des dynamiques techniques, économiques et de travail, entre innovation de situations nouvelles et préservation de la flexibilité face aux aléas.

\section{Encadré 6. Trois profils d'organisation du travail (Cournut et al 2010).}

L'étude porte sur 19 fermes réparties dans quatre régions du monde (Pampa d'Argentine et d'Uruguay, Amazonie brésilienne, Ségala français, montagnes du nord du Vietnam) et choisies avec nos partenaires locaux de recherche et développement.

L'analyse comparative combine des variables relatives aux caractéristiques structurelles et productives des élevages et d'autres décrivant la configuration du collectif de travail, l'aménagement des bâtiments et équipements, la conduite de l'élevage et des surfaces ainsi que l'ajustement des activités non agricoles.

Trois profils d'organisation, peu liés à la région, se différencient par le recours à l'entraide, au salariat permanent et par la simplification de la conduite du troupeau et/ou des surfaces.

\section{Les simplificateurs (6 exploitations)}

Ce profil est caractérisé par la simplification de la conduite du troupeau et/ou des surfaces, et le recours à l'entraide pour le travail de saison sur les surfaces. Le travail lié au troupeau est réalisé par le noyau organisateur de façon assez autonome.

Les entrepreneurs (7 exploitations)

Tous ces exploitants emploient au moins un salarié permanent. Leurs conduites techniques sophistiquées sont exigeantes en travail d'astreinte et/ou de saison du fait du développement des cultures, de l'entretien des surfaces ou de l'abondance des stocks.

Les autonomes ( 6 exploitations)

Le noyau organisateur est composé d'au moins deux personnes (couples ou associés) et ces exploitants recourent rarement à d'autres formes de main-d'œuvre. Les conduites ne présentent pas de caractère particulier au regard de la variabilité observée dans l'échantillon. 


\section{Références}

Agabriel J., Farrié J.P., Pottier E., Note P., Pomiès D., 2012. Conséquences zootechniques de simplifications de pratiques : exemples de la distribution des aliments et de la traite des vaches. In : Numéro spécial, Travail en élevage. Hostiou N., Dedieu B., Baumont R. (Eds). INRA Prod. Anim. 25, 141-158.

Allaire G., 1988. Itinéraires et identités professionnelles des travailleurs de l'agriculture, INRA Actes et communications, 3, 175-211.

Altieri M.A., 1992. Agroecological foundations of alternative agriculture in California. Agric., Ecosyst. Env. 39, 23-53.

Attonaty J.M., Chatelin M.H., Poussin J.C., Soler L.G., 1990. Un simulateur à base de connaissance pour raisonner équipement et organisation du travail en agriculture. In : Econ. Artif. Intel., Bourgine P., Walliser B. (Eds), Paris, France, 291-297.

Aubry C., Bressoud F., Petit C., 2011. Les circuits courts en agriculture revisitent-ils 1'organisation du travail dans l'exploitation ? Réflexions sur les enjeux d'une prise en compte. In : Beguin P., Dedieu B., Sabourin E. (Eds) «le travail en agriculture : son organisation et ses valeurs face à l'innovation». Editions L'harmattan, Paris, France, 19-35.

Bagès R., Rieu-Gout A., 1981. Conditions de travail et mode de vie des éleveurs de brebis laitières du sud Aveyron. Econ. Rurale, 142, p4.

Barthez A., 1986. Du labeur paysan au métier d'agriculteur. L'élaboration statistique en agriculture. Cah. Econ. Sociol. Rurales, 3, 45-72.

Beguin P., 2011. Réflexions sur les enjeux d'une prise en compte du travail agricole dans la conception d'une agriculture durable. In : Beguin P., Dedieu B., Sabourin E. (Eds) «le travail en agriculture : son organisation et ses valeurs face à l'innovation». Editions L'harmattan, Paris, France, 37-52.

Beguin P., Dedieu B., Sabourin E., (Eds), 2011. Le travail en agriculture : son organisation et ses valeurs face à l'innovation. Editions L'Harmattan, Paris, France, 304 p.

Bellet V., 1999. Le travail lié à la surface fourragère dans les systèmes herbagers ovins de Poitou-Charentes. Fourrages 160, 359-371.

Billon P., Pomiés D., 2006. Le point sur la robotisation de la traite 15 ans après l'apparition des premiers systèmes dans les fermes. Renc. Rech. Rum., 13, 143-150.

Bishoff O., Balard J., Pin A., Chauvat S. Dumonthier P., Serviere G., Dedieu B., 2008. L'organisation du travail en élevage. Enseigner la méthode Bilan Travail. Supagro Florac/INRA, Institut de l'Elevage, Educagri Editions, Dijon, France, 154 p.

Blanchemanche S., 2000. La combinaison d'activités professionnelles des ménages agricoles. L'exemple du département de l'Isère. Thèse, Université de Paris, France, 468p.

Boivin X., Bensoussan S., L'hotellier N., Bignon L., Brives H., Brulé A., Godet J., Grannec M.L., Hausberger M., Kling-Eveillard F., Tallet C., Courboulay V., 2012. Hommes et animaux d'élevage au travail : vers une approche pluridisciplinaire des «pratiques relationnelles». In : Numéro spécial, Travail en élevage. Hostiou N., Dedieu B., Baumont R. (Eds). INRA Prod. Anim., 25, 159-168.
Brangeon J.L., Jégouzo G., de Roze B., 1988. La durée annuelle du travail professionnel agricole en élevage laitier et porcin. In Emploi et conditions de travail dans l'agriculture et l'agroalimentaire. INRA, Actes et Communications, 3, 31-43.

Brookfield H., 2008. Family farms are still around: time to invert the old agrarian question. Geography Compass, 2-1, 108-126.

Calland B., Madelrieux S., Pauthenet Y., 2009. Changements et Organisation du Travail (COTE) en Exploitation d'élevage-Alpes du Nord. In : Recueil d'outils et démarches de conseil sur le travail en élevage. Document Institut de l'Elevage, http://www.idele.fr

Cellier J.M., Marquié J.C., 1980. Systèmes d'activité et régulations dans l'exploitation agricole. Le Travail Humain, 43, 321-336.

Cerf M., Sagory P., 2004. L'agriculture, un secteur critique dans les sociétés. In : Falzon P. (Ed). «traité d'ergonomie», Editions PUF, Paris, France, 491-499.

Chabanet G., Dedieu B., Serviere G., 1999. Cohérences entre la répartition du travail du salariat au sein du groupement d'employeurs et la fonction qu'il remplit chez un adhérent. Etude de trois cas en Auvergne, Renc. Rech. Rum., 6, 81-84.

Charroin T., Veysset P., Fromont J.L., Devienne S., Palazon R., Ferrand M., 2012 Productivité du travail et économie en élevages d'herbivores : Définition des concepts, analyse et enjeux. In : Numéro spécial, Travail en élevage. Hostiou N., Dedieu B., Baumont R. (Eds). INRA Prod. Anim., 25, 193-210.

Chauvat S., 2010. Référentiel travail en élevages ovins viande. Synthèse de 137 Bilans Travail. Document Institut de l'Elevage, http://www.idele.fr

Chauvat S., Sabatté N., 2009. Recueil d'outils et démarches de conseil sur le travail en élevage. Document Institut de l'Elevage, http://www.idele.fr

Cittadini R., Dedieu B., Derail L., Perez R., 2001. Trabajo y tecnología en explotacionesganaderas de la provincia de Buenos Aires. In Neiman G. (Ed). "Trabajo de campo. Produccion, technologia y empleo en el medio rural”. Ediciones Ciccus, 120-133.

Correa P., Dieguez F., Dedieu B., Arbeletche P., Bartaburu D., Moralés H., Tourrand.F 2011. Comprendre l'organisation du travai pour préciser les stratégies d'éleveurs laitiers uruguayens. In : Beguin P., Dedieu B., Sabourin E. (Eds), «Le travail en agriculture son organisation et ses valeurs face à l'innovation». Editions L'harmattan, Paris, France, 135154

Coulon J.B., Lecomte P., 2009. Dispositif de recherches INRA-CIRAD sur les productions animales en régions chaudes : contexte, état des lieux et perspectives. In : Enjeux et priorités de la recherche pour l'élevage dans les pays du Sud. Session satellite, Renc. Rech. Rum., 43-50.

Cournut S., Chauvat S., 2012. L'organisation du travail en exploitation d'élevage : analyse de 630 Bilans Travail réalisés dans huit filières animales. In : Numéro spécial, Travail en élevage. Hostiou N., Dedieu B, Baumont R. (Eds). INRA Prod. Anim., 25, 101-112.

Cournut S., Jordan A., Dedieu B., Servière G., 2009. Analyse de groupe des Bilans Travail.
Guide méthodologique. Document Institut de l'Elevage, http://www.idele.fr

Cournut S., Serviere G., Hostiou N., Chauvat S., Dedieu B., 2010. L'organisation du travail en exploitations familiales d'élevage. Enseignements d'une analyse comparée conduite en France, en Amérique latine et au Vietnam. Cah. Agric., 19, 338-347, 1-10.

Commandeur M., 2006. Diversity of pig farming styles: understandinghow it is structured. Neth. J. Agric. Sci., 54, 111-127.

Crawford A., Nettle R., Brightling B., Hibburt C., 2010. Enhancing the liveability of farm systems: Supporting new farm and advisory practices in the Australian dairy industry. Proc. Eur. IFSA Symp., Vienna, Austria. http://ifsa.boku.ac.at/cms/fileadmin/Proceedin g2010/2010 WS2.3 Crawford.pdfpp

Curie J., Hajjar V., 1987. Vie de travail, vie hors travail : la vie en temps partagé. In Lévy-Leboyer C., Sperandio J.C., Traité de psychologie du travail, 37-55.

Darnhofer I., Bellon S., Dedieu B., Milestad R., 2009. Adaptiveness to enhance the sustainability of farming systems. A review. Agron. Sus. Dev., 30, 545-555.

Darnhofer I., Gibbon D., Dedieu B., 2012. Farming Systems Research: an approach to inquiry. In: Darnhofer, Gibbon D. ; Dedieu B. (Eds) "Farming Systems Research into the 21st century: The new dynamic". Springer, 1-30. Sous presse.

Dedieu B., 1984. L'élevage ovin sur parcours méditerranéens. Adaptation et mutations des systèmes de production en Cévennes gardoises. Thèse INA Paris-Grignon, France, 302p.

Dedieu B., 1993. Organisation du travail et fonctionnement d'exploitations d'élevage extensif du Massif Central. Etud. Rech. Syst. Agraires. Dev., 27, 303-322.

Dedieu B., Serviere G., 2001. Organisation du travail et fonctionnement des systèmes d'élevage. Renc. Rech. Rum., 8, 245-250.

Dedieu B., Serviere G., 2011. Les modèles du travail en élevage : points de vue de zootechniciens des systèmes d'élevage. In : Beguin P., Dedieu B., Sabourin E. (Eds) «Le travail en agriculture: son organisation et ses valeurs face à l'innovation». Editions L'harmattan, Paris, France, 155-170.

Dedieu B., Chabanet G., Josien E., Becherel F., 1997. Organisation du pâturage et situations contraignantes en travail : démarche d'étude et exemples en élevage bovin viande. Fourrages, 149, 21-36.

Dedieu B., Chauvat S., Servière G., Tchakérian E., 2000. Bilan travail pour l'étude du fonctionnement des exploitations d'élevage. Institut de l'Elevage, http://www.idele.fr

Dedieu B., Faverdin P., Dourmad J.Y., Gibon A., 2008a. Système d'élevage, un concept pour raisonner les transformations de l'élevage. In : Numéro spécial, 20 ans de recherches en productions animales à l'INRA. Charley B. Herpin P., Perez J.M. (Eds). INRA Prod. Anim., 21, 45-58.

Dedieu B., Chia E., Leclerc B., Moulin C.H., Tichit M. 2008b. L'élevage en mouvement : flexibilité et adaptation des exploitations d'herbivores. Coll. Sci. Technol., Update. Editions Quae, Paris, France, 294p. 
Depoudent C., Le Moan L., 2011 Déterminants de la fidélisation d'une équipe de salariés en élevage porcin. Journ. Rech. Porcine, 43, 255-256.

Dejours C., 1998 Travailler n'est pas déroger Travailler, 1, 5-12.

Dufour A., Dedieu B., 2010. Rapports au temps de travail et modes d'organisation en élevage laitier. Cah. Agric., 19, 377-382.

Dufour A., Giraud C., 2012.Le travail dans les exploitations d'élevage bovin laitier est-il toujours conjugal ? In : Numéro spécial, Travail en élevage. Hostiou N., Dedieu B., Baumont R. (Eds). INRA Prod. Anim., 25, 169-180.

Fagon J., Sabatte N., 2010. Référentiel travail en élevages bovins lait. Synthèse de 190 Bilans Travail. Document Institut de l'Elevage, http://www.idele.fr

Fiorelli C., 2010. L'aménagement des conditions de vie au travail des éleveurs : proposition d'un cadre d'analyse des relations entre rapport subjectif et organisation du travail en élevage. Etude de cas chez les éleveurs pluriactifs. Agro ParisTech., France, 300p.

Fiorelli C., Porcher J., Dedieu B., 2007. Pourquoi faire de l'élevage quand on a un autre travail ? Renc. Rech. Rum., 14, 389-392.

Fiorelli C Dedieu B, Porcher J., 2010. Proposition d'un cadre d'analyse des compromis réalisés par les éleveurs pluriactifs pour organiser leur travail. Cah. Agric., 19, 383-390.

Fiorelli C., Mouret S., Porcher J., 2012. Les rationalités du travail avec les animaux d'élevage : produire, vivre ensemble et se construire. In : Numéro spécial, Travail en élevage. Hostiou N., Dedieu B, Baumont R. (Eds). INRA Prod. Anim., 25, 181-192.

Gras C., Hernandez V., 2007. Changement du modèle productif et acteurs sociaux dans le paysage rural argentin. In : Hernandez V., OuldAhmed P., Papail J., Phélinas P. (Eds). L'action collective à l'épreuve de la globalisation. Editions L'Harmattan, Paris, France, 47-80.

Havet A., Joly N., Cochet A., 2005. Les écrits comme supports de raisonnement dans les exploitations d'élevage. Renc. Rech. Rum., $12,327-330$

Hémidy L., 1986. Organisation du travail en salle de traite. Une approche par simulation. Bull. Tech. Inf. Min. Agr., 412-413,657-678.

Hémidy L., Maxime F., Soler L.G., 1993. Instrumentation et pilotage stratégique dans l'entreprise agricole. Cah. Econ. Sociol. Rurales, 28, 33-50.

Hervé D., Genin D., Migueis J., 2002. A modelling approach for analysis of agro-pastoral activity at the one-farm level. Agric. Syst. $71,187-206$

Hostiou N., Dedieu B., 2009. Diversity of forage system work and adoption of intensive techniques in dairy cattle farms of Amazonia. Agron. Sust. Dev., 29, 535-544.

Hostiou N., Dedieu B., 2011. A method for assessing both work productivity and flexibility in livestock farms. Animal, doi:10 1017/S1751731111002084.

Hostiou N., Fagon J., 2012. Simplification des conduites d'élevage : analyse transversale des pratiques mises en œuvre dans les filières herbivores et granivores. In : Numéro spécial, Travail en élevage. Hostiou N., Dedieu B., Baumont R. (Eds). INRA Prod. Anim., 25, $127-140$
Hostiou N., Dedieu B., Madelrieux S. DuyKhanh P., Binh Vu T., 2010. Relation entre organisation du travail et taille des exploitations laitières : une étude à Moc Chau (Vietnam) Cah. Agric., 19, 323-330.

IGER, 1977. Temps de travaux dans les ateliers de production ovine. Doc IGER-BCMEAITOVIC, 243p.

IFSA, 2010. (European Group of the International Farming Systems Association).Site internet de l'association http://ifsa.boku.ac.at

Ingrand S., Dedieu B., 1996. Diversité des formules d'allotement en élevage bovin viande. Le cas d'exploitations du Limousin. INRA Prod. Anim., 9, 189-199.

Jordan A., Servière G., Journal C., Dedieu B., Chauvat S., 1996. Bilan des bilans-travail Séminaire «Bilan-travail dans les exploitation d'élevage», ENITA de Clermont-Ferrand, France, 28p.

Josien E., Dedieu B., Chassaing C., 1994. Etude de l'utilisation du territoire en élevage herbager. L'exemple du réseau extensif bovin Limousin. Fourrages, 138, 115-134.

Kling-Eveillard F., Cerf M., Chauvat S. Sabatté N., 2012, Le travail, sujet intime et multifacette : premières recommandations pour l'aborder dans le conseil en élevage. In Numéro spécial, Travail en élevage. Hostiou N., Dedieu B., Baumont R. (Eds). INRA Prod. Anim., 25, 211-220.

Lacroix A., Mollard A., 1991. Mesurer le travail agricole : de l'enregistrement à la reconstitution analytique, Cah. Econ. sociol. Rurales, 20, 27-46.

Landais E., Deffontaines J.P., 1988. Les pratiques des agriculteurs. Points de vue sur un courant nouveau de la recherche agronomique. Etudes Rurales, 109, 125-158.

Laurent C., Cartier S., Fabre C., Mundler P. Ponchelet S., Remy J. 1998. L'activité agricole des ménages ruraux et la cohésion économique et sociale. Econ. Rurale, 244, 12-21.

Laurent C., Blanchemanche S., Hassani F., Mundler P., 2000. Une nouvelle économie du temps pour les ménages agricoles. INRA, Symposium de Montpellier «Recherche pour et sur le développement territorial», tome 1, 227-241.

Lokhorst C., Groot Koerkamp P.W.P., 2009. Precision livestock farming '09.Wageningen University press, $368 \mathrm{p}$.

Lémery B., 2003. Les agriculteurs dans la fabrique d'une nouvelle agriculture. Sociologie du travail, 45, 9-25.

Leplat J., 1994. Collective activity in work: some ways of research. Le travail humain, 57 , 209-226.

Lorino P., 1995. Comptes et récits de la performance. Essai sur le pilotage de l'entreprise. Editions d'organisation, Paris, France. 288p.

Madelrieux S., Dedieu B., 2008. Qualification and assessment of work organisation in livestock farms. Animal, 2-3, 435-447.

Madlerieux S., Dedieu B., Dobremez L. 2006. ATELAGE : un modèle pour qualifier l'organisation du travail dans les exploitations d'élevage, INRA Prod. Anim., 19, 47-58.

Madlerieux S., Dedieu B., Dobremez L., Girard N., 2009. Patterns of work organisation in livestock farms: the ATELAGE approach Livest. Sci., 121, 28-37.
Mak S., 2001.Continued innovation in a Cambodian rice-based farming system: farmer testing and recombination of new elements. Agric. Systems, 69, 137-149.

Martel G., Dedieu B., Dourmad J.Y., 2007. Do labour productivity and preferences about work load distribution affect reproduction management and performance in pig farms? Livest. Sci., 116, 96-107.

Martel G., Dedieu B., Dourmad J.Y., 2008. Simulation of sow herd dynamics with emphasis on performance and distribution of periodic tasks events. J. Agric. Sci., 146, 365-380.

Milestad R., Dedieu B., Darnhofer I., Bellon S., 2012. Farms and farmers facing change: the adaptive approach. In : Darnhofer I., Gibbon D., Dedieu B. (Eds) "Farming Systems Research into the 21st century: The new dynamic". Springer, 367-388. Sous presse.

Meuret M., 2010. Un savoir-faire de bergers. Educagri et Editions Quae, Paris, France, 336p.

Mintzberg H., 2002. Structure et dynamique des organisations. Editions d'Organisation, Paris, France.

Moneyron A., 2003. Transhumance et écosavoirs. Editions L'Harmattan, Paris, France, 236p.

Muchagata M., Brown K., 2003. Cows, colonists and trees: rethinking cattle and environmental degradation, Agric. Syst. 76, $797-$ 816.

Nicourt C., Cabaret J, 2011. Création de normes, innovation sanitaire et éthique des éleveurs ovins bio. In : Beguin P., Dedieu B., Sabourin E. (dir) «Le travail en agriculture : son organisation et ses valeurs face à l'innovation». Editions L'harmattan, Paris, France, 8598.

Paillard S., Treyer S., Dorin B., (Eds). 2011. Agrimonde. Scénarios and challenges for feeding the world in 2050. Editions Quae, Versailles, France, 295p.

Papy F., Attonaty J.M., Laporte C., Soler L.G., 1988. Work organization simulation as a basis for farm management advice (equipment and manpower, levels against climatic variability). Agric. Syst., 27, 295-314

Peyraud J.L., Baumont R., 2010. Programme coopératif de recherche et développement sur les systèmes de production animale à hautes performances économiques et environnementales. GIS Elevages demain. 20p.

Pichereau F., Becherel F., Farrié JP., Legendre J., Veron J., Lequenne D., Mage C. Serviere G., Cournut S., Dedieu B., 2004 Fonctionnement des grands troupeaux de vaches allaitantes : analyse des déterminants structurels et techniques de l'organisation du travail. Renc. Rech. Rum., 11, 129-136.

Porcher J., 2002. Eleveurs et animaux : réinventer le lien. Editions PUF, Paris, France, $204 p$.

Porcher J., 2011 Vivre avec les animaux, une utopie pour le 21 ème siècle. Editions La Découverte, Paris, France, $168 \mathrm{p}$

Rault G., 2005. Approche sociologique du travail en élevage et questions sur le conseil agricole, in Séminaire AFPF, Elevage, prairies, travail, 29-35.

Rémy J., 2008. «Paysans, exploitants familiaux, entrepreneurs...» : de qui parlons-nous ? Coll. international de l'Association Française de Science Politique : Les Mondes Agricoles 
en politique, Centre Etudes Rech. Int., Paris, France, 6p.

Salmona M., 1994. Les paysans français : le travail, les métiers, la transmission des savoirs. Editions L'harmattan, Paris, France, 371p.

Sarzeaud P., Chauvat S., 2010. Travibov, Démarche de conseil sur le travail en élevage bovin - Guide méthodologique. Document Institut de l'Elevage, http://www.idele.fr

Sébillotte M., 1986. Evolution et actualité des problèmes d'organisation du travail en agriculture. Bull. Tech. Inf. Min. Agric., 412413, 621-630

Seegers J., Moreau J.C., Béguin E., Guillaumin A., Frappat B., 2006. Attentes des éleveurs laitiers vis-à-vis de leurs conditions de travail et évolution de leurs systèmes d'exploitation. Fourrages, 185, 3-16.

Soriano V., 1985. Choisir réussir son élevage ou le roman de la technique et de la passion. Doc. Ministère Agriculture, 64p.
Valax MF., 1989. La gestion du temps dans l'exploitation agricole. B.T.I., 345-52.

Véron F., Dobremez L., 2004. Impact des Opérations Locales agri-environnementales et de la «prime à l'herbe» sur les prairies des zones de montagne. Fourrages, 177, 25-48.

Vincent I, Houi S., Pineau C., 2011 Utilisation des références en études supérieures courtes agricoles. In : Travailler mieux en élevage. Conférence au Space. Document Institut de l'Elevage. http://www.idele.fr

\title{
Résumé
}

Les mutations du travail en élevage sont structurelles car les tailles des exploitations augmentent continuellement. Elles sont aussi sociologiques car les éleveurs souhaitent de meilleures conditions de vie au travail. Elles sont également liées aux innovations technologiques visant une plus grande efficience des pratiques. Les éleveurs intègrent l'ensemble de ces mutations en adaptant les conduites des troupeaux et des surfaces ainsi que les autres éléments de l'organisation du travail (la main-d'œuvre, les équipements). Pour décrire et qualifier les formes d'organisation du travail en élevage, évaluer leur efficience et leur flexibilité, les zootechniciens des systèmes d'élevage ont proposé, avec des emprunts à l'économie, à l'ergonomie et à l'agronomie, des modèles qui considèrent que ni les travaux, ni les travailleurs, ni les périodes de l'année ne sont équivalents. Ils visent à mettre en relation les différentes facettes de l'agriculteur, à la fois manager technico-économique, organisateur du travail et travailleur sensible. Ces propositions reprises par les conseillers en France et à l'étranger contribuent à la constitution d'une communauté de recherche, formation et développement autour de ce thème stratégique pour l'avenir de l'élevage.

\begin{abstract}
Twenty years of research and development on work in livestock farming: achievements and prospects

Transformations in work with livestock are structural, due to the increasing size of farms. They are sociological: livestock farmers want to have better working conditions. Technologies that aim at better efficiencies push changes in work as well. Livestock farmers integrate all these transformations and adapt their herd and land area managements while reorganizing the other components of work organization (equipment and workforce). The work models proposed by livestock scientists, with borrowings from economics, ergonomics, and agronomy, consider that neither the work nor the workers, nor the periods of the year are equivalent. They try to connect the different facets of the farmer's work (that of the technico-economical manager, work organizer and sensitive operator) and make it possible to qualify work organization and assess its efficiency and flexibility. The propositions have been taken up by advisors in France and abroad. They contribute to the emerging community of research, extension and education which has been created over 20 years around this theme, which is strategic for the future of livestock farming.
\end{abstract}

DEDIEU B., SERVIÈRE G., 2012. Vingt ans de recherche - développement sur le travail en élevage : acquis et perspectives. In : Numéro spécial, Travail en élevage. Hostiou N., Dedieu B., Baumont R. (Eds). INRA Prod. Anim., 25, 85-100. 